This item was submitted to Loughborough's Research Repository by the author.

Items in Figshare are protected by copyright, with all rights reserved, unless otherwise indicated.

\title{
Efficiency analysis with ratio measures
}

PLEASE CITE THE PUBLISHED VERSION

http://dx.doi.org/10.1016/j.ejor.2015.03.013

\section{PUBLISHER}

(C) Elsevier B.V.

\section{VERSION}

AM (Accepted Manuscript)

\section{PUBLISHER STATEMENT}

This work is made available according to the conditions of the Creative Commons Attribution-NonCommercialNoDerivatives 4.0 International (CC BY-NC-ND 4.0) licence. Full details of this licence are available at: https://creativecommons.org/licenses/by-nc-nd/4.0/

\section{LICENCE}

CC BY-NC-ND 4.0

\section{REPOSITORY RECORD}

Olesen, Ole Bent, Niels Christian Petersen, and Victor Podinovski. 2015. "Efficiency Analysis with Ratio Measures". Loughborough University. https://hdl.handle.net/2134/18163. 


\title{
Efficiency Analysis with Ratio Measures
}

\author{
Ole Bent Olesen ${ }^{\mathrm{a}}$, Niels Christian Petersen ${ }^{\mathrm{a}}$, Victor V. Podinovski ${ }^{\mathrm{b}, *}$ \\ ${ }^{a}$ Department of Business Economics, The University of Southern Denmark, Campusvej 55, DK-5230 \\ Odense M, Denmark \\ ${ }^{b}$ Warwick Business School, University of Warwick, Coventry CV4 7AL, UK
}

\begin{abstract}
In applications of data envelopment analysis (DEA) data about some inputs and outputs is often available only in the form of ratios such as averages and percentages. In this paper we provide a positive answer to the long-standing debate as to whether such data could be used in DEA. The problem arises from the fact that ratio measures generally do not satisfy the standard production assumptions, e.g., that the technology is a convex set. Our approach is based on the formulation of new production assumptions that explicitly account for ratio measures. This leads to the estimation of production technologies under variable and constant returns-to-scale assumptions in which both volume and ratio measures are native types of data. The resulting DEA models allow the use of ratio measures "as is", without any transformation or use of the underlying volume measures. This provides theoretical foundations for the use of DEA in applications where important data is reported in the form of ratios.
\end{abstract}

Keywords: data envelopment analysis, technology, production assumptions, efficiency, ratio measures

\section{Introduction}

Analysis of efficiency of organizations is an important area of management science which can be approached by different methodologies. Data envelopment analysis (DEA) is a wellestablished non-parametric methodology used for the assessment of efficiency of organizations, referred to as decision making units (DMUs) (Cooper et al., 2007; Thanassoulis et al., 2008). From the economic perspective, DEA makes use of a multidimensional model of production technology in which each dimension represents an input or output. The technology extrapolates the observed set of DMUs based on stated production assumptions. The efficiency of DMUs is measured relative to the efficient (non-dominated) frontier of the production technology.

One of the long-standing methodological questions in DEA is whether ratio measures are acceptable as input and output data. Such measures can be found in many applications of DEA and include various percentages, proportions, rates and averages. These are usually obtained as the ratio of two underlying volume measures, although more complex ratio measures are not uncommon.

Ratio measures are often used as contextual variables, for example, to represent the quality of the socio-economic environment in which DMUs operate, such as income per capita,

\footnotetext{
*Corresponding author

Email addresses: ole@sam.sdu.dk (Ole Bent Olesen), ncp@sam.sdu.dk (Niels Christian Petersen), victor.podinovski@wbs.ac.uk (Victor V. Podinovski)
} 
unemployment rate, average age and proportion of people on benefits in a community. Contextual ratio measures may also represent inputs that are non-discretionary for management at the lower level of organizations, such as marketing costs per capita.

Ratio measures are also often used to represent attributes of the inputs or outputs used in the analysis. In the case of outputs, these may reflect the quality of products and services, such as success rates in health care, pass rates in academic exams and average time for graduates to find a job. Ratio measures may also represent the performance of a production or service process, for example, the proportion of services delivered within a target lead time. In the case of inputs, ratio measures often characterize their quality, for example, the proportion of students with good academic record on entry to schools.

In some applications ratio measures represent inputs or outputs directly, and not as a complement to volume measures. For example, performance analysis of the financial sector often focuses on such ratios as the equity capital to asset ratio, net charge-offs to loans and return on assets.

The suitability of ratio data in DEA models has been subject of academic debate for some time, although much of this has not been properly documented. The earlier discussion focused on the acknowledgement of the problems that ratio data presented to DEA and attempted to decide which of the standard DEA models were more suitable for such datasee, e.g., Golany and Thore (1997), Dyson et al. (2001), Hollingsworth and Smith (2003), and Cooper et al. (2007, page 19).

\subsection{Motivational example}

We use the following simple example to illustrate the problems with ratio measures in DEA applications. Examples similar to this could be found in most sectors, from education to banking, but we choose hospital management for two reasons. First, this sector gives us a variety of ratios of different types in a single setting. Second, most readers should be intuitively familiar with its context and terminology.

Example 1. Consider a decision maker (DM) who wishes to assess the performance of a group of publicly funded hospitals. Suppose the purpose of this assessment is to identify the treatment costs of the efficient hospitals and use these as benchmarks in funding decisions.

To keep our discussion focused, suppose the DM wishes to use a DEA model with a single aggregate volume input (costs), and two aggregate volume outputs (inpatients and outpatients). ${ }^{1}$ In line with the literature on health care applications of DEA, the DM may use either the variable or constant returns-to-scale (VRS or CRS) model. The choice depends on whether the DM believes that scale economies cannot be ignored (and use VRS) or are negligible (and assume CRS).

Suppose that the DM wishes to take into account the following additional inputs and outputs represented by ratio measures:

$\mathcal{R}_{1}$ : success rate for certain treatments;

$\mathcal{R}_{2}$ : proportion of patients with a certain condition treated within the target time;

$\mathcal{R}_{3}$ : acute readmission rates from primary admissions in selected diagnostic categories;

$\mathcal{R}_{4}$ : mortality rates after specific surgical procedures;

$\mathcal{R}_{5}$ : rate of cancer radiotherapy treatments per device (number of treatments per hour, per device);

$\mathcal{R}_{6}$ : the average distance from the hospital to the patients;

\footnotetext{
${ }^{1}$ We also assume that the hospitals are sufficiently homogeneous, and that all performance measures are adjusted for age, gender, casemix and other relevant factors.
} 
Table 1: Hospitals in Example 2.

\begin{tabular}{cccc}
\hline Hospital & Patients & Successful treatments & Success rate \\
\hline$A$ & 1000 & 200 & 20 \\
$B$ & 500 & 400 & 80 \\
$C=0.5 A+0.5 B$ & 750 & 300 & 50 (incorrect), 40 (correct) \\
\hline
\end{tabular}

$\mathcal{R}_{7}$ : proportion of the population in the catchment area with specific adverse health conditions such as diabetes or obesity;

$\mathcal{R}_{8}$ : proportion of the population in the catchment area who have received vaccination against flu (funded separately by state-sponsored programs).

The dilemma that the DM faces is: what DEA model should be used?

Below, in the rest of this introductory section, we first show that neither the conventional VRS nor CRS model is appropriate for the above situation - this is because the use of ratio measures makes them theoretically unsubstantiated and efficiency rankings arbitrary. We then review the relevant literature on the subject and show that the few suggested approaches are insufficient or unsatisfactory for the above example. Finally, we use this example to motivate the new models developed in our paper and outline the logic of our approach.

We give an answer to the stated DM's dilemma at the end of our paper in $\S 8$.

\subsection{Why are ratio measures problematic in the VRS and CRS DEA models?}

Banker et al. (1984) state the production assumptions (axioms) assumed true in the standard CRS and VRS production technologies. If some of these axioms are not satisfied, the DEA model of production technology becomes an arbitrary extension of the observed data set, and the analysis based on it is generally unsubstantiated.

It is straightforward to show that the use of ratio measures is generally inconsistent with such production assumptions, most importantly the assumption that the technology is a convex set in all input and output dimensions. This was first shown by Olesen and Petersen $(2006,2009)$ and further discussed by Emrouznejad and Amin (2009). The following example, based on Olesen and Petersen (2006), demonstrates this point.

Example 2. Consider two hospitals, $A$ and $B$, whose outputs are shown in Table 1 . The first output is the number of patients who have received a certain treatment. The second output is the number of successful treatments. The last column shows the success rate for the treatment calculated as the ratio of the second to the first output (ratio $\mathcal{R}_{1}$ in Example 1). Let $C$ be the simple average of hospitals $A$ and $B$, that is, their convex combination taken with equal weights of 0.5 . Hospital $C$ has 750 patients of which 300 were successfully treated, and its success rate is $40 \%$. Note that this is less than $50 \%$, which is the simple average of the success rates of $20 \%$ and $80 \%$ of hospitals $A$ and $B$. It is clear that the $50 \%$ success rate is unsubstantiated. This problem arises from the fact that the denominators in the two ratios are different (1000 for hospital $A$ and 500 for hospital $B$ ).

In the CRS model, ratio measures are also generally inconsistent with the assumption of proportionality that allows proportional scaling of its inputs and outputs. As an illustration, refer to Example 1. If the volume inputs and outputs (costs and patients) are scaled in a certain proportion, the ratios from $\mathcal{R}_{1}$ to $\mathcal{R}_{6}$ may also change, but not in the same proportion 
or even direction as the volume measures. Furthermore, ratios $\mathcal{R}_{7}$ and $\mathcal{R}_{8}$ are exogenous and should remain constant regardless of the scaling.

The above shows that the use of ratio measures in the standard VRS and CRS models generally results in an incorrect model of the production technology. ${ }^{2}$

It is sometimes argued that the efficiency obtained in the VRS and CRS models is still meaningful and can be defined using their dual multiplier forms. The latter do not explicitly refer to the notion of technology and define efficiency through the ratio of total weighted output to input. An obvious drawback of this approach is that the efficiency defined only through the multiplier model lacks its technological meaning as a possible improvement factor for inputs or outputs (because the technology as the set of production possibilities is not correctly modeled). Even the radial nature of efficiency in the standard VRS and CRS models is not obvious from the multiplier formulations. Furthermore, such important notions as returns to scale, scale efficiency, and productivity change become unsubstantiated.

While the above interpretation may be useful in some contexts, it does not help the DM in Example 1: because the multiplier model does not model the technology, the benchmarks (including costs) for inefficient hospitals obtained from this model are not necessarily achievable, and funding decisions based on the use of such benchmarks would be unsubstantiated and possibly flawed.

\subsection{Existing alternative approaches}

While acknowledging problems with ratio data, the existing DEA literature suggests few approaches that can be used to overcome them, and such approaches have important limitations.

\subsubsection{Using volume measures instead of ratios}

It is often suggested that one should use the underlying volume measures (used as the numerator and denominator in ratios) - see, e.g., Thanassoulis et al. (1995) and Emrouznejad and Amin (2009). This approach is attractive but may not be possible or practical, or even appropriate, for a number of reasons.

First, the underlying volume data may be unavailable. Second, the use of volume measures may make little sense in the given context. For example, consider the use of income per capita in the catchment area as a contextual ratio measure linked to student attainment in the assessment of school performance. Neither the numerator (total income) nor the denominator (population of the area) would be considered relevant in such models. Third, the use of ratio measures may be embedded in the organizational decision making, and the analyst may be required to use such data even if the volume data is available - see, e.g., Golany and Thore (1997). Fourth, the volume data may be judged to be too sensitive and confidential, so the ratio data has to be used instead.

\footnotetext{
${ }^{2} \mathrm{~A}$ simple exception to this is a re-normalization of a particular volume input or output by the same denominator for all DMUs. For example, suppose the annual production level (output) is changed to the average monthly production level, by dividing the former by twelve. Because the denominator is the same for all DMUs, it is straightforward to verify that such a re-normalization does not affect the validity of the VRS and CRS production assumptions. For the same reason, changing the units of measurement, e.g., currency, used in the measurement of inputs or outputs does not lead to any problems with the production assumptions.

In the case of CRS (but not VRS) another obvious exception is the normalization of all inputs and outputs of each DMU by a single unit-specific denominator. For example, in a comparative assessment of countries, all inputs and outputs of each country may be divided by its population (or all divided by its GDP). This re-scaling does not change the CRS technology. However, if different measures are divided by different country-specific denominators (e.g., some by the population and some by GDP), the convexity assumption is no longer satisfied and the conventional CRS technology becomes unsubstantiated.
} 


\subsubsection{Using free disposal hull (FDH) model}

The FDH model (Deprins et al., 1984) does not assume convexity and can in principle be used with both volume and ratio measures. However, because this model does not use the information that volume inputs and outputs can form convex combinations (and can be scaled in the case of CRS), the resulting technology would be unnecessarily small, and the efficiency scores overestimated. In practice this often manifests itself in low efficiency discrimination between DMUs, especially when the observed sample is small.

\subsubsection{Other approaches}

Dyson et al. (2001) suggest that a ratio measure could be multiplied by an appropriate measure of the size of the DMU, so that the resulting measure could change in proportion to the size of the latter. An obvious drawback of this approach is that any measure, except the denominator used in the ratio in the first place, is arbitrary.

Close to this discussion are studies of the use of environmental and categorical variables in DEA that raise arguments equally applicable to socio-economic types of ratio data (Banker and Morey, 1986a, 1986b; Ruggiero 1996). For similar types of contextual data the approaches of Paradi et al. (2004b), Paradi et al. (2009), and Olesen and Petersen (2009) aim at limiting the use of DMUs operating in a favorable environment in benchmarks for DMUs operating in a harsh environment. The transformation of outputs representing categorical quality measures to related cumulative volume measures before their incorporation in a CRS model is explored in Olesen and Petersen (1995).

\subsection{Contribution of this paper}

The main contribution of this paper is the development of Ratio-VRS and Ratio-CRS (R-VRS and R-CRS) models that allow the incorporation of ratio measures for inputs and outputs "as is", without any transformation of the data. This gives a constructive answer to the long-standing debate as to how ratio measures could be used in DEA models.

The idea of our approach is that ratio measures should not be incorporated in the conventional DEA models: the latter have been developed for volume measures that exhibit different characteristics compared to ratio measures. Instead, we obtain new DEA models in which both volume and ratio measures are native types of data.

The following specific contributions should be mentioned. First, we revisit the conventional production axioms and restate them in the forms that account for ratio measures: this includes new variants of the axioms of convexity and proportionality.

Second, we suggest that in the R-CRS model, ratio measures should be represented differently according to the way in which they respond to the scaling of volume measures. For example, increasing the size of a hospital (or the number of treatments) may improve the success rate $\mathcal{R}_{1}$ (Gaynor et al., 2005) and possibly reduce the mortality rate $\mathcal{R}_{4}$, while keeping the contextual ratios $\mathcal{R}_{7}$ and $\mathcal{R}_{8}$ constant. To address this diversity of behavior, we develop a classification of ratio measures into different types and suggest their functional representations.

Third, we formally derive the new production technologies from the stated axioms. A particularly complex task accomplished in our paper is the development of a unifying $R-C R S$ model that incorporates ratio measures of all types, in one single formulation.

Fourth, we explore theoretical properties of the new DEA models, discuss computational approaches to their solution and illustrate our development by an example. 


\section{Basic definitions and notation}

A basic assumption of most applications of DEA is that the observed DMUs are elements of some production technology $T$. While the mainstream DEA is concerned with the model specification that has a clear economic and technological sense, in many applications of DEA the latter is not considered essential or even required (Paradi et al., 2004a; Dulá, 2009). Even if $T$ has a clear technological meaning, it may be desirable to incorporate additional ratio measures whose economic meaning may be questionable but which have an obvious managerial or policy meaning, such as the average salary increase after obtaining a degree or life expectancy after a hospital treatment.

The main focus of our development is on the use of ratio data in technologies that have a clear economic interpretation. Our results also extend to any application beyond the traditional economic boundaries, provided the technology $T$ (perhaps, better called $a$ correspondence set in such cases) satisfies all the assumptions stated below.

For a production technology $T$, let $\mathrm{I}=\{1, \ldots, m\}$ and $\mathrm{O}=\{1, \ldots, s\}$ be the sets of inputs and outputs, respectively. Let $\mathrm{I}^{V} \subseteq \mathrm{I}$ and $\mathrm{O}^{V} \subseteq \mathrm{O}$ be the subsets of volume inputs and outputs (volume measures). The complementary subsets $\mathrm{I}^{R}=\mathrm{I} \backslash \mathrm{I}^{V}$ and $\mathrm{O}^{R}=\mathrm{O} \backslash \mathrm{O}^{V}$ include, respectively, ratio inputs and outputs (ratio measures).

It is important to underline that, when modeling production technologies, volume and ratio measures are classed as inputs or outputs depending on the way they satisfy the assumption of free disposability, stated as Axiom 2 below. Inputs have a nonnegative impact on production, and their larger values (compared to the current level of a DMU) are assumed to be technologically possible. Outputs have a nonpositive impact in the sense that their smaller values are technologically possible. In the case of ratio measures, their classification into inputs and outputs often has no direct relationship to what types of volume measure (input or output) are specified as the numerator and denominator. For example, in the assessment of school performance, each of the following would be a volume output of its own (because each consumes resources, and smaller numbers are technologically possible in the sense of free disposability): $y_{1}$-all students, $y_{2}$-students with special needs, and $y_{3}$ - students from privileged background. Note that, from the technology point of view, the ratio $y_{2} / y_{1}$ is an output (smaller values are technologically possible), while $y_{3} / y_{1}$ is an input (larger values are possible).

Elements of $T$ are decision making units, or DMUs. These are stated in the form

$$
(X, Y)=\left(X^{V}, X^{R}, Y^{V}, Y^{R}\right)
$$

where $X \in \mathbb{R}_{+}^{m}$ and $Y \in \mathbb{R}_{+}^{s}$ are the vectors of inputs and outputs, respectively, and the subvectors $X^{V}, X^{R}, Y^{V}$ and $Y^{R}$ correspond to the sets $\mathrm{I}^{V}, \mathrm{I}^{R}, \mathrm{O}^{V}$ and $\mathrm{O}^{R}$. Observed DMUs are denoted $\left(X_{j}, Y_{j}\right)$, where $j \in J=\{1, \ldots, n\}$.

In standard DEA models no specific upper bounds are imposed on inputs and outputs. This assumption may clearly be incorrect for ratio measures. Therefore, we assume that there are upper bounds in the form

$$
X^{R} \leq \bar{X}^{R} \text { and } Y^{R} \leq \bar{Y}^{R}
$$

where components of vectors $\bar{X}^{R}$ and $\bar{Y}^{R}$ can be finite or $+\infty .^{3}$ The latter means that no particular bound is specified on the corresponding ratio measure. For example, the

\footnotetext{
${ }^{3}$ We use vector inequalities to state that the specified inequality is true for each component of the vector. For example, $X^{R} \leq \bar{X}^{R}$ means $X_{i}^{R} \leq \bar{X}_{i}^{R}$, for all $i \in \mathrm{I}^{R}$.
} 
proportion of satisfied customers is naturally bounded above by $100 \%$, while the firm's growth rate is not and can be more than $100 \% .{ }^{4}$

The following two assumptions are required for the development of the R-VRS technology with ratio measures in the next section.

Data Assumption 1. At least one of the two sets I and $O$ is not empty. ${ }^{5}$

Data Assumption 2. For all $j \in J$, observed DMUs $\left(X_{j}, Y_{j}\right) \in \mathbb{R}_{+}^{m} \times \mathbb{R}_{+}^{s}$ and their subvectors of ratio measures $X_{j}^{R}$ and $Y_{j}^{R}$ satisfy (2).

\section{The R-VRS technology}

\subsection{Axioms}

As discussed above, ratio measures cannot be assumed to satisfy the standard axioms of the VRS technology, and the axioms need restating. The first two are straightforward.

Axiom 1 (Feasibility of observed data). For any $j \in J,\left(X_{j}, Y_{j}\right) \in T$.

Axiom 2 (Free disposability). Let $(X, Y) \in T$. If $(\tilde{X}, \tilde{Y}) \in \mathbb{R}_{+}^{m} \times \mathbb{R}_{+}^{s}$ satisfies the bounds (2), $\tilde{X} \geq X$ and $\tilde{Y} \leq Y$, then $(\tilde{X}, \tilde{Y}) \in T$.

As a motivation of the third axiom, refer to the above Example 2. As noted, the incorrectness with the convex combinations arises if the ratio measures have different denominators for different DMUs. An obvious exception from this is when the DMUs have equal ratios, even if the denominators are different. For example, if both hospitals have the same $80 \%$ success rate then, regardless of the number of patients, the average of the two hospitals (hospital $C$ ) has an $80 \%$ success rate. This means that convex combinations of DMUs in the presence of ratio data are still possible provided their ratio inputs and outputs take on respectively equal values.

The above observation corresponds to the notion of selective convexity introduced by Podinovski (2005) who extended earlier ideas explored by Banker and Morey (1986b) and Ruggiero (1996). In the context of volume and ratio measures, this observation is stated as follows. ${ }^{6}$

Axiom 3 (Selective convexity). Let $(\tilde{X}, \tilde{Y}) \in T$ and $(\hat{X}, \hat{Y}) \in T$. Assume that

$$
\tilde{X}^{R}=\hat{X}^{R} \text { and } \tilde{Y}^{R}=\hat{Y}^{R} \text {. }
$$

Then $\gamma(\tilde{X}, \tilde{Y})+(1-\gamma)(\hat{X}, \hat{Y}) \in T$, for any $\gamma \in[0,1]$.

\footnotetext{
${ }^{4}$ Bounds (2) are needed for the formal definition of technologies based on ratio measures. They may also be binding (and cannot be omitted) in special cases of DEA models based on general directional distance function (Chambers et al., 1998). However, these bounds are automatically satisfied and need not to be stated as explicit constraints in most common cases when the input or output radial efficiency is assessed. This is stated in Proposition 7.

${ }^{5}$ Note that Data Assumption 1 is somewhat unusual because it allows one of the sets I or O to be empty. The standard assumption that there is at least one input and at least one output is not needed for the R-VRS technology, although this changes in the R-CRS technology, as stated in Data Assumption 4 in $\S 5$. This may be of interest to applications of DEA in which there are no clear inputs or outputs. In such cases a common approach is to introduce a dummy input or output equal to one for all DMUs. In view of this comment, this is redundant.

${ }^{6}$ In the development of the R-CRS technology in $\S 4$, we introduce different types of ratio measures. Axiom 3 assumes that condition (3) is satisfied by any type of ratio measure.
} 


\subsection{Definition of the R-VRS technology}

Based on the stated production axioms, and in line with the minimum extrapolation principle used by Banker et al. (1984), we give the following definition:

Definition 1. The R-VRS technology $T_{\mathrm{VRS}}^{\mathrm{R}}$ is the intersection of all technologies (sets) $T \subset \mathbb{R}_{+}^{m} \times \mathbb{R}_{+}^{s}$ that satisfy Axioms 1-3.

The rationale of the above definition is as follows. While there exist infinitely many technologies that satisfy Axioms 1-3, technology $T_{\mathrm{VRS}}^{\mathrm{R}}$ is the smallest among all of them. ${ }^{7}$ This guarantees that $T_{\mathrm{VRS}}^{\mathrm{R}}$ includes only the DMUs that are needed to satisfy the stated axioms, and no arbitrary DMUs.

To obtain an explicit statement of technology $T_{\mathrm{VRS}}^{\mathrm{R}}$, we use a related formulation proved in Podinovski (2005) for the technology based on the notion of selective convexity. In comparison to the latter result, the following theorem accounts for bounds (2) on ratio measures. $^{8}$

Theorem 1. Let Data Assumptions 1 and 2 be true. Technology $T_{\mathrm{VRS}}^{\mathrm{R}}$ is the set of all DMUs $(X, Y) \in \mathbb{R}_{+}^{m} \times \mathbb{R}_{+}^{s}$ that satisfy (2) and for which there exists a vector $\lambda \in \mathbb{R}^{n}$ such that

$$
\begin{aligned}
& \sum_{j \in J} \lambda_{j} Y_{j}^{V} \geq Y^{V}, \\
& \sum_{j \in J} \lambda_{j} X_{j}^{V} \leq X^{V}, \\
& \lambda_{j}\left(Y_{j}^{R}-Y^{R}\right) \geq \mathbf{0}, \\
& \lambda_{j}\left(X_{j}^{R}-X^{R}\right) \leq \mathbf{0}, \\
& \mathbf{1}^{\top} \lambda=1 \\
& \lambda \geq \mathbf{0} .
\end{aligned}
$$

The meaning of conditions (4) is intuitively clear. If $\lambda_{j}>0$, inequalities (4.3) and (4.4) imply $Y_{j}^{R} \geq Y^{R}$ and $X_{j}^{R} \leq X^{R}$, respectively. This means that the observed DMUs used in the convex combinations of volume inputs and outputs in (4.1) and (4.2) are not worse than the DMU $(X, Y)$ on all ratio inputs and outputs. To be more specific and expanding the logic of Ruggiero (1996), let ratio measures represent the quality of the socio-economic environment in which the DMUs operate or the quality of services they provide. Then the volume outputs and inputs of the DMU $(X, Y)$ are weakly outperformed (weakly dominated) by a convex combination of observed DMUs that operate in the same or worse environment, or produce services of the same or higher quality than $(X, Y)$.

Proposition 1. Let Data Assumptions 1 and 2 be true. Then technology $T_{\mathrm{VRS}}^{\mathrm{R}}$ is a closed set.

If there are no ratio measures and $\mathrm{I}^{V} \neq \varnothing$ and $\mathrm{O}^{V} \neq \varnothing$, inequalities (4.3) and (4.4) vanish and $T_{\mathrm{VRS}}^{\mathrm{R}}$ becomes the standard VRS technology. If there are no volume measures and $\mathrm{I}=\mathrm{I}^{R}$ and $\mathrm{O}=\mathrm{O}^{R}$, then $T_{\mathrm{VRS}}^{\mathrm{R}}$ becomes $\mathrm{FDH}$. If $\mathrm{O}^{R}=\varnothing, T_{\mathrm{VRS}}^{\mathrm{R}}$ is the technology used in model (7) in Ruggiero (1996).

Although all conditions (4) are linear with respect to $\lambda$, the technology $T_{\mathrm{VRS}}^{\mathrm{R}}$ is not a convex set. Figure 1 shows technology $T_{\text {VRS }}^{\mathrm{R}}$ induced by two DMUs $A$ and $B$. It has a volume

\footnotetext{
${ }^{7}$ It is straightforward to verify that the technology $T_{\mathrm{VRS}}^{\mathrm{R}}$ satisfies Axioms 1-3 and bounds (2).

${ }^{8}$ We use bold symbols $\mathbf{0}$ and $\mathbf{1}$ for the vectors whose components are all zeros and ones, respectively. The dimensions of these vectors are clear from the context. The symbol ${ }^{\top}$ means transposition.
} 


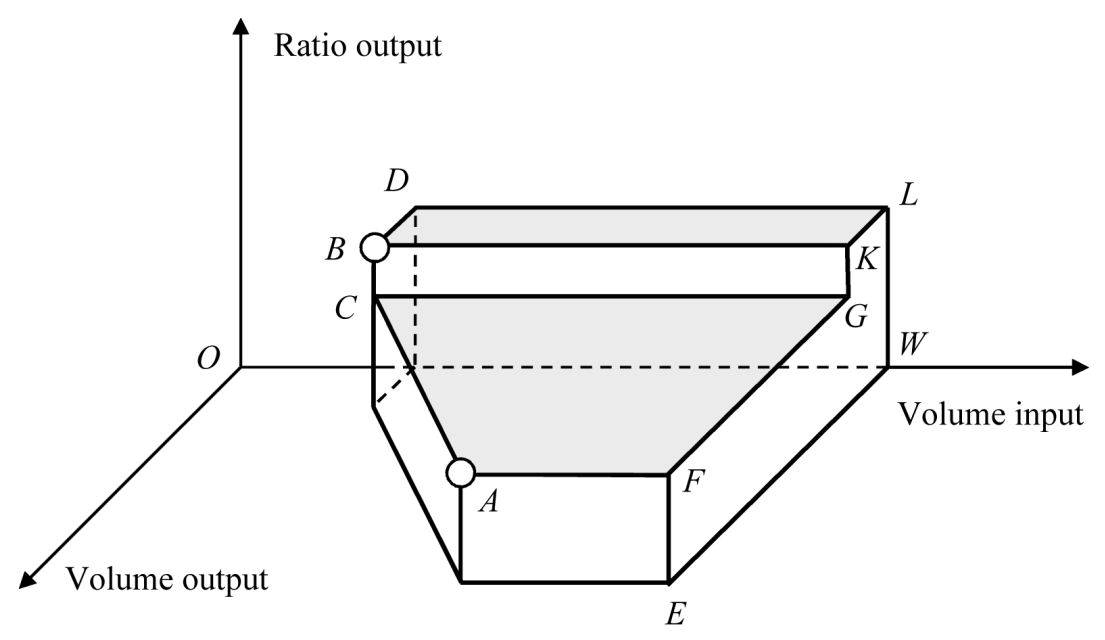

Figure 1: Technology $T_{\mathrm{VRS}}^{\mathrm{R}}$ with a ratio output.

input, volume output and a ratio output. This technology is unbounded on the right: its section EFGKLW shows its nonconvex section for a fixed level $W$ of the volume input. Note that any section of $T_{\mathrm{VRS}}^{\mathrm{R}}$ for a fixed level of ratio output is convex.

\subsection{Treatment of bad inputs and outputs}

Axiom 2 provides an unambiguous classification of volume and ratio measures into inputs and outputs. This leads to a straightforward treatment of bad (undesirable) outputs (such as the readmission and mortality rates $\mathcal{R}_{3}$ and $\mathcal{R}_{4}$ in Example 1 ) and bad inputs (such as the proportion $\mathcal{R}_{7}$ of people with adverse health conditions). If larger values of bad outputs are possible in the sense of Axiom 2, these are (technological) inputs that are modeled by inequalities (4.2) or (4.4), as any other input measure. Similarly, if bad inputs are classified by Axiom 2 as (technological) outputs, they satisfy output inequalities (4.1) or (4.3). This modeling issue is separate from the question whether, for example, in the calculation of input radial efficiency, the improvement factor should be attached only to the good inputs or to the bad outputs (modeled as inputs) as well. ${ }^{9}$

\section{Types of ratio measures under the assumption of CRS}

The standard CRS technology $T_{\mathrm{CRS}}$ (Charnes et al., 1978) is defined by the axioms of VRS and the additional assumption of proportionality (Banker et al., 1984). Consider any DMU $\left(X^{*}, Y^{*}\right) \in T_{\mathrm{CRS}}$, where $X^{*}$ and $Y^{*}$ are the vectors of volume inputs and outputs, respectively. The assumption of proportionality states that, for any scaling factor $\alpha \geq 0$, the DMU $\left(\alpha X^{*}, \alpha Y^{*}\right) \in T_{\mathrm{CRS}}$. Obviously, this assumption does not extend to the majority of ratio measures.

In order to define an R-CRS technology with ratio measures, we need to specify how each ratio measure changes in response to the proportional scaling of the volume measures with $\alpha \geq 0$.

\footnotetext{
${ }^{9}$ There is also an extensive literature which deals with the treatment of bad outputs and inputs that cannot be assumed to satisfy Axiom 2 (as either input or output) and are instead assumed to satisfy the property of weak disposability. Our development excludes this type of volume and ratio measures, and leaves it open for future research.
} 
Consider, for example, a simple scenario with a single ratio (input or output) measure $\mathcal{R}$. Then any DMU $\mathcal{D}$ is represented by the triplet $\left(X^{*}, Y^{*}, z^{*}\right)$, where $z^{*}$ is the value of ratio $\mathcal{R}$ observed at the DMU. If the volume measures are scaled by $\alpha \geq 0$, the resulting DMU can be stated as $\mathcal{D}(\alpha)=\left(\alpha X^{*}, \alpha Y^{*}, z(\alpha)\right)$, where $z=z(\alpha)$ describes $\mathcal{R}$ as a function of $\alpha$. Obviously, $z(1)=z^{*}$. Our task is to specify the function $z=z(\alpha)$ such that the DMU $\mathcal{D}(\alpha)$ is technologically possible (producible) for any $\alpha \geq 0$.

Below we show that most ratio inputs and outputs arising in practical applications (including all ratio measures from $\mathcal{R}_{1}$ to $\mathcal{R}_{8}$ in Example 1 ) can be modeled by a small number of simple linear and piecewise-linear functions $z=z(\alpha)$. Based on this, we classify ratio measures into different types. We use this classification to state the R-CRS technology in which each ratio measure is modeled differently, according to its type. ${ }^{10}$

A particular problem that we have to address in our development is that the exact response of the ratio measure $\mathcal{R}$ to the scaling factor $\alpha$ is often unknown, although it is usually possible to assume that the measure is non-decreasing or non-increasing in $\alpha$. In such cases it is impossible to estimate the actual ratio $\mathcal{R}$ as a function of $\alpha$. To overcome this problem, we define $z=z(\alpha)$ as the most conservative and safe bound on the ratio $\mathcal{R}$ based on the worst-case assumptions.

\subsection{Fixed ratio measures}

Fixed ratio inputs and outputs can be assumed constant while the volume measures are scaled up or down with $\alpha \geq 0$ :

$$
z(\alpha)=z^{*}, \quad \forall \alpha \geq 0 .
$$

Two distinct groups of ratios would normally be classed as being of the fixed type.

Contextual measures. These include uncontrollable (non-discretionary) socio-economic and environmental factors. In Example 1, $\mathcal{R}_{8}$ is a non-discretionary (for hospital management) fixed ratio input. Similarly, $\mathcal{R}_{7}$ is a bad input modeled as fixed ratio output. In other assessment contexts further examples include income per capita (ratio input), academic ability of students on entry (ratio input), proportion of school children eligible for some form of support, and unemployment rate in the area (both are undesirable inputs modeled as ratio outputs).

Quality factors obtained as the ratios of two mutually proportional volume measures. The idea is that if both volume measures are changed in the same proportion, their ratio remains constant. The success rate of hospital treatments (ratio $\mathcal{R}_{1}$ in Example 1 ) is a possible example of a fixed ratio output. However, if existing evidence suggests that $\mathcal{R}_{1}$ is an increasing function of the number of treatments (Gaynor et al., 2005), a more appropriate model for this ratio output is the downward-proportional type discussed below. In the educational context, an example of fixed ratio output is the pass rate in exams that may be assumed invariant of the size of the school.

\subsection{Proportional ratio measures}

Proportional ratio measures change in the same proportion $\alpha$ as the volume measures:

$$
z(\alpha)=\alpha z^{*}, \quad \forall \alpha \geq 0
$$

\footnotetext{
${ }^{10}$ It should be noted that the suggested types do not include all theoretically possible ratio measures. For example, ratio measures modeled by non-monotone functions $z(\alpha)$ are not covered by our classification and cannot be used in the developed models of technology. The main criterion for deciding which types of ratio measures to include in this paper has been whether or not we could identify relevant examples such as $\mathcal{R}_{1}-\mathcal{R}_{8}$ in the motivational example.
} 
Measures of this type are typically the ratios of a controllable volume output or input to a constant volume measure. When the numerator changes in proportion $\alpha$ and the denominator remains constant, the ratio changes in the same proportion $\alpha{ }^{11}$

Although proportional ratios respond to $\alpha$ in the same way as volume measures, the former do not satisfy the assumption of convexity and require a different treatment.

Example 3 (Proportional ratio output). In Example 1, the average distance from the hospital to patients $\mathcal{R}_{6}$ is a bad input modeled as a ratio output. Large average distances imply long travel times to the hospital. For certain illnesses, like cardiac arrest, long transportation may aggravate the patient's condition and imply a more complicated (and costly) treatment. As the size of hospital increases, so does its catchment area and the average distance to the patients, although the exact relationship is impossible to specify. In this case formula (6) provides a reasonable approximation and classes $\mathcal{R}_{6}$ as a proportional ratio output.

Example 4 (Proportional ratio input and output). Applications of DEA to the assessment of policy performance of nations (viewed as DMUs) usually involve various macroeconomic, social factors and policy variables. The transformation of inputs to outputs in these models is regarded not as a result of a production process but rather as a result of different government policies (Golany and Thore, 1997). The data available to such applications is often given in the form of ratios (e.g., normalized per capita or as a proportion of GDP), growth rates, etc.

The assumption of CRS in the above context imply proportional relationship between the ratio input and output measures. Examples include GDP per capita (ratio input) and education expenditure per capita (ratio output). Note that that the denominator (population of the country) is different for different DMUs. In this context, GDP per capita should be regarded as a proportional ratio input, and education expenditure per capita as a proportional ratio output.

\subsection{Downward-proportional ratio outputs}

This modeling type is suitable for ratio outputs $\mathcal{R}$ that are non-decreasing functions of $\alpha$. This includes the cases where the exact functional form is either unknown, or is known but the fixed or proportional types do not describe $\mathcal{R}$ sufficiently well. Figure 2 illustrates this type. Point $A$ represents ratio $\mathcal{R}$ at $\alpha=1$. Because we do not know the actual curve $U A W$ or its shape is too complex, we model $\mathcal{R}$ by its lower bound represented by the broken line $B A C$.

Using output-specific notation $y=y(\alpha)$ instead of the generic function $z=z(\alpha)$, the downward-proportional ratio output ${ }^{12}$ is modeled as follows:

$$
y(\alpha)= \begin{cases}\alpha y^{*}+(1-\alpha) q, & \text { if } 0 \leq \alpha<1, \\ y^{*}, & \text { if } \alpha \geq 1\end{cases}
$$

The value $y(\alpha)$ of this type remains constant for $\alpha \geq 1$ and is reduced in a linear fashion as $\alpha$ decreases from 1 to 0 , from the value $y(1)=y^{*}$ to some assumed value $q \in\left[0, y^{*}\right]$. Because the true ratio $\mathcal{R}$ is intentionally underestimated by the function $y(\alpha)$ in $(7)$ and $\mathcal{R}$ is a ratio output, the resulting $\operatorname{DMU} \mathcal{D}(\alpha)=\left(\alpha X^{*}, \alpha Y^{*}, y(\alpha)\right)$ must be technologically possible for all $\alpha \geq 0$.

\footnotetext{
${ }^{11}$ Formula (6) applies to unbounded ratio measures. We deal with bounded ratio measures at the end of this section.

${ }^{12}$ In our naming convention, the word "downward-proportional" refers to the scaling factor $\alpha$ being reduced from the value $\alpha=1$ downward to 0 , and not to the decreasing or increasing type of the function $y(\alpha)$. In contrast, the term "upward-proportional" used in $\S 4.5$ means the proportionality on the right of $\alpha=1$.
} 


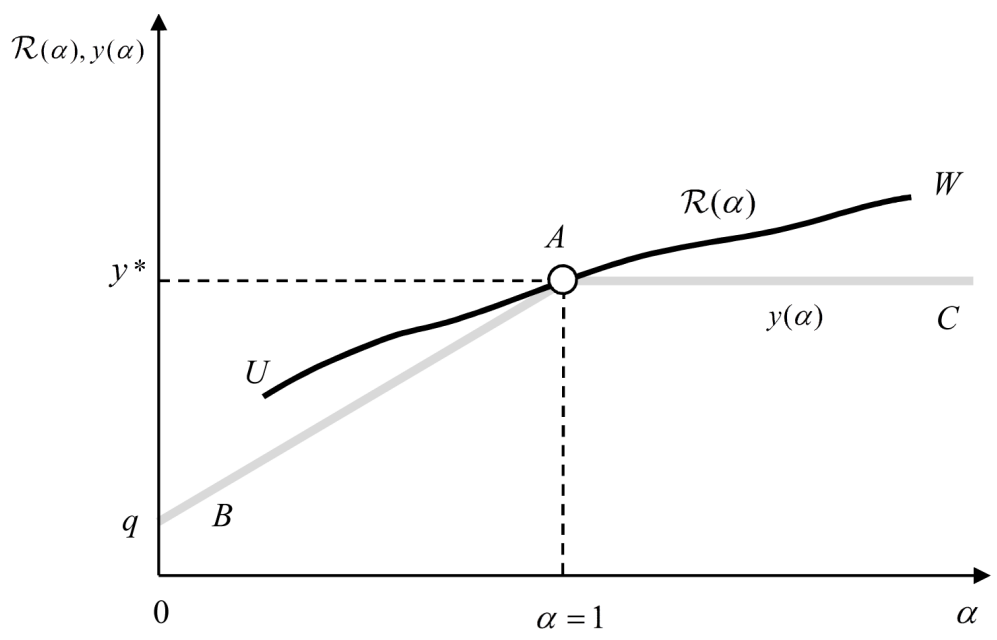

Figure 2: Downward-proportional ratio output $\mathcal{R}=\mathcal{R}(\alpha)$ and its lower bound $y=y(\alpha)$. The curve $U A W$ shows the actual (possibly unknown) ratio output which is non-decreasing in $\alpha$. It is conservatively modeled by its lower bound $y(\alpha)$ represented by line $B A C$.

Example 5 (Downward-proportional ratio output). Measures $\mathcal{R}_{1}$ and $\mathcal{R}_{2}$ from Example 1 may be regarded as ratio outputs of this type. To be specific, consider $\mathcal{R}_{1}$, the success rate for a certain treatment as a function of $\alpha$. Empirical evidence (Gaynor et al., 2005) suggests that the success rate for some treatments may improve with the larger number of treatments. In line with this, if $\alpha>1, \mathcal{R}_{1}$ may increase, but the exact relationship is difficult to specify. In the worst case, $\mathcal{R}_{1}$ should remain at least at the level $y^{*}$. If $\alpha<1$, it is reasonable to assume that $\mathcal{R}_{1}$ does not decrease below the value $\alpha y^{*}+(1-\alpha) q$, where $q$ is an assumed worst possible success rate for the given treatment. In the absence of any information about $q$ it is always possible to take $q=0$. Formula (7) describes these scenarios and models $\mathcal{R}_{1}$ as a downward-proportional ratio output.

\subsection{Downward-proportional ratio inputs}

Similar to its ratio output analogue, this type is suitable for the modeling of ratio inputs $\mathcal{R}$ that are non-increasing functions of $\alpha$, as represented by the curve $U A W$ in Figure 3. If the exact relationship between $\mathcal{R}$ and $\alpha$ cannot be assumed to be of the fixed type or is unknown, we model $\mathcal{R}$ by its upper bound represented by the broken line $B A C$.

Using input-specific notation $x=x(\alpha)$, this type can be described as follows:

$$
x(\alpha)= \begin{cases}\alpha x^{*}+(1-\alpha) p, & \text { if } 0 \leq \alpha<1, \\ x^{*}, & \text { if } \alpha \geq 1,\end{cases}
$$

where $p \geq x^{*}$ is an assumed value. Because the function $x(\alpha)$ in (8) overestimates the ratio input $\mathcal{R}$, the $\mathrm{DMU} \mathcal{D}(\alpha)=\left(\alpha X^{*}, \alpha Y^{*}, x(\alpha)\right)$ must be technologically possible for all $\alpha \geq 0$.

Example 6 (Downward-proportional ratio input). Let us show that ratios $\mathcal{R}_{3}$ and $\mathcal{R}_{4}$ from Example 1 may be viewed as measures of this type. Both measures are bad outputs, and we treat them as ratio inputs. To be specific, consider $\mathcal{R}_{4}$, the mortality rate after a specific surgery procedure as a function of $\alpha$. Because, as pointed in Example 5, the success rate may increase with the number of treatments, it is reasonable to assume that $\mathcal{R}_{4}$ is a nonincreasing function of $\alpha$. Therefore, if $\alpha>1$, the mortality rate $\mathcal{R}_{4}$ cannot exceed $x^{*}$. To model the case $\alpha<1$, we need to specify (assume) the worst-case upper bound $p \geq x^{*}$ on 


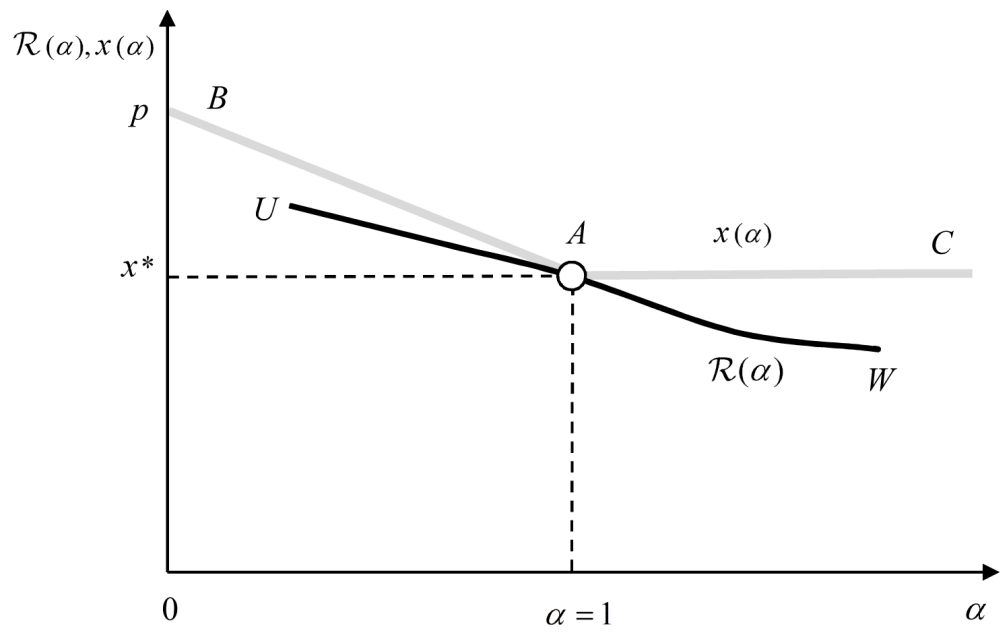

Figure 3: Downward-proportional ratio input $\mathcal{R}=\mathcal{R}(\alpha)$ and its upper bound $x=x(\alpha)$. The curve $U A W$ shows the actual (possibly unknown) ratio input which is non-increasing in $\alpha$. It is conservatively modeled by its upper bound $x(\alpha)$ represented by line $B A C$.

the mortality rate for the given surgical procedure. If no estimate is available, we can use the logically highest value $p=1$. It may then be reasonable to assume that the line $B A$ in Figure 3 represents a conservative upper bound on all values $\mathcal{R}_{4}$ if $0 \leq \alpha<1$, as stated by formula (8).

\subsection{Upward-proportional ratio inputs}

This type can be used to model ratio inputs $\mathcal{R}$ that are non-decreasing functions of $\alpha$. Figure 4 illustrates this type. If the exact functional form of $\mathcal{R}$ is unknown or does not allow the use of fixed or proportional input types, we model $\mathcal{R}$ by its upper bound shown as the broken line $B A C$.

This type is formally described by the function $x(\alpha)$ that is constant for $0 \leq \alpha \leq 1$ and increases proportionally for $\alpha>1$ :

$$
x(\alpha)= \begin{cases}x^{*}, & \text { if } 0 \leq \alpha<1 \\ \alpha x^{*}, & \text { if } \alpha \geq 1\end{cases}
$$

Example 7 (Upward-proportional ratio input). Consider the rate of radiotherapy treatments at a hospital per device (ratio $\mathcal{R}_{5}$ in Example 1). The higher capacity of radiotherapy facilities at larger hospitals (with larger number of devices) allows for well-known advantages from pooling queues potentially resulting in lower waiting times and higher throughput per device. Therefore, it may be reasonable to assume that $\mathcal{R}_{5}$ is a non-decreasing function of $\alpha$.

We may view $\mathcal{R}_{5}$ as a ratio input because lower waiting times (corresponding to higher values of $\mathcal{R}_{5}$ at larger hospitals) contribute to speedier treatment of patients and higher success rates. Because $\mathcal{R}_{5}$ is non-decreasing in $\alpha$, it cannot exceed the constant value $x^{*}$ in the case $0 \leq \alpha<1$. For $\alpha>1$ we might expect $\mathcal{R}_{5}$ to increase but not in proportion exceeding $\alpha$. Formula (9) describes these two cases and models $\mathcal{R}_{5}$ as an upward-proportional ratio input.

\subsection{Bounded ratio measures}

Below we consider ratio measures that have a finite upper bound specified by conditions (2). For the fixed or downward-proportional types, formulae (5), (7) and (8) keep the ratios 


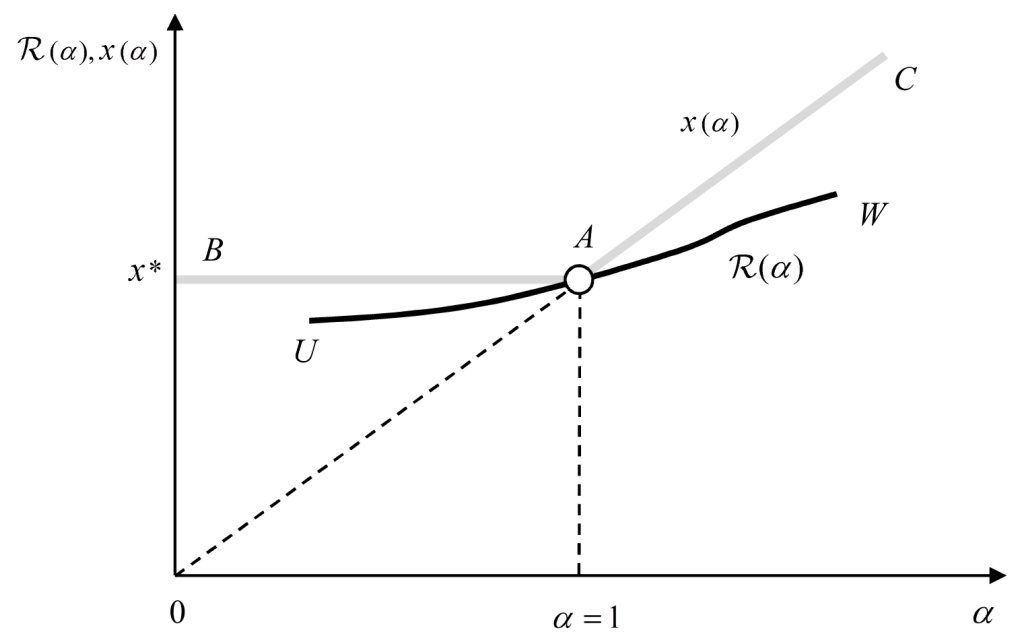

Figure 4: Upward-proportional ratio input $\mathcal{R}=\mathcal{R}(\alpha)$ and its upper bound $x=x(\alpha)$. The curve $U A W$ shows the actual (possibly unknown) ratio input which is non-decreasing in $\alpha$. It is conservatively modeled by its upper bound $x(\alpha)$ represented by line $B A C$.

within the bounds (2) and, therefore, require no adjustment. The proportional and upwardproportional types in (6) and (9) are, however, inconsistent with the finite upper bounds and should be reconsidered.

Bounded Proportional Ratio Outputs. In the context of socio-economic performance of countries described in Example 4, the enrolment ratio for secondary education $y=y(\alpha)$ may be viewed as a bounded ratio output which is proportional with respect to GDP per capita (treated as ratio input). ${ }^{13}$ The ratio $y(\alpha)$ satisfies (6) but only until it reaches the upper bound of 1, and remains constant if $\alpha$ continues to increase. Formula (6) for a proportional ratio output $y(\alpha)$ with an upper bound $\bar{y}$ takes on the form:

$$
y(\alpha)=\min \left\{\alpha y^{*}, \bar{y}\right\}, \quad \forall \alpha \geq 0 .
$$

Bounded Proportional and Upward-Proportional Ratio Inputs. In the assessment of competitiveness of nations, the proportion of population with a certain level of education $x(\alpha)$ may be viewed as a proportional ratio input. Note that $x(\alpha)$ can be assumed to satisfy (6) only until it reaches the upper bound of $\bar{x}=1$. A further increase of $\alpha$ implies increasing other proportional (e.g., volume) measures in proportion $\alpha$ while keeping the ratio $x(\alpha)=1$ constant. This is hardly acceptable because the numerator (volume input) of $x(\alpha)$ is no longer increasing. Therefore an upper bound $\bar{x}$ on ratio $x(\alpha)$ puts an upper bound $\bar{\alpha}=\bar{x} / x^{*} \geq 1$ on $\alpha$ for which the R-CRS technology is defined. ${ }^{14}$

\footnotetext{
${ }^{13}$ The enrolment ratio is obtained by dividing the number of students enroled in secondary school education by the total number of young people of the corresponding age.

${ }^{14}$ The following example provides an alternative argument why $\alpha$ should not exceed the value $\bar{\alpha}=\bar{x} / x^{*}$. Let $\left(X^{*}, Y^{*}, x^{*}\right)$ be an observed unit, where $X^{*}$ and $Y^{*}$ are the vectors of volume inputs and outputs, and $x^{*}$ is a proportional ratio input with an upper bound $\bar{x}=1$. Let $x^{*}=0.5$. If $\alpha$ is unbounded, applying $\alpha_{1}=4$ and noting the upper bound on $x$, the observed unit becomes $\left(4 X^{*}, 4 Y^{*}, 1\right)$. We now apply $\alpha_{2}=0.25$ to the resulting unit to obtain $\left(X^{*}, Y^{*}, 0.25\right)$. The latter dominates the original observed unit. This means that no observed unit with $x^{*}>0$ can be fully efficient, which is meaningless in a practical application. Note that the same example does not apply if $x^{*}$ is an upward-proportional ratio input. However, it seems inconsistent and, therefore, unacceptable to allow $\alpha$ exceed the value $\bar{x} / x^{*}$ for upward-proportional ratio inputs and disallow this for proportional inputs. As proved in Proposition 7 , the specification of bounds $\bar{x}$ is primarily of theoretical interest and affects only a limited number of practical models of efficiency analysis.
} 


\section{The R-CRS technology}

In this section we develop an extension to the standard CRS technology that allows the incorporation of ratio inputs and outputs. This is based on Axioms 1-3 and two further axioms.

\subsection{Additional notation and axioms}

Banker et al. (1984) define the CRS technology as the one based on the same axioms as the VRS technology and the additional axiom of proportionality, or "ray unboundness". Below we maintain the latter property with respect to volume measures but change it with respect to ratio measures, taking into account the types of ratio measures introduced in $\S 4$.

Let, as above, $\mathrm{I}^{V}$ and $\mathrm{O}^{V}$ be the sets of volume inputs and outputs, respectively, and let $X^{V}$ and $Y^{V}$ be the corresponding vectors of volume inputs and outputs. Similarly, let superscripts $F, D, U$ and $P$ refer to the four types of ratio measures: fixed, downwardproportional, upward-proportional and proportional, respectively, as defined in $\S 4$. For example, we denote $\mathrm{I}^{F}$ and $\mathrm{O}^{F}$ the sets of fixed ratio inputs and outputs, respectively, and denote $X^{F}$ and $Y^{F}$ the corresponding vectors. DMUs $(X, Y)$ are stated in the following extended form that replaces (1):

$$
\left(\begin{array}{c}
X \\
Y
\end{array}\right)=\left(\begin{array}{c}
X^{V}, X^{F}, X^{D}, X^{U}, X^{P} \\
Y^{V}, Y^{F}, Y^{D}, Y^{P}
\end{array}\right)
$$

Similarly, the upper bounds in (2) are stated as $\bar{X}^{R}=\left(\bar{X}^{F}, \bar{X}^{D}, \bar{X}^{U}, \bar{X}^{P}\right)$ and $\bar{Y}^{R}=$ $\left(\bar{Y}^{F}, \bar{Y}^{D}, \bar{Y}^{P}\right)$.

For downward-proportional ratio outputs $r \in \mathrm{O}^{D}$, the constant $q$ in (7) may be different for different $r$. Denote $\bar{q}$ the vector of dimension $\left|\mathrm{O}^{D}\right|$ whose components $\bar{q}_{r} \geq 0$ are equal to the corresponding constants $q$. Similarly, define the vector $\bar{p}$ of dimension $\left|\mathrm{I}^{D}\right|$ whose components $\bar{p}_{i} \geq 0$ are equal to the corresponding constants $p$ specified in (8) for the ratio inputs $i \in \mathrm{I}^{D}$.

Consider any DMU $(X, Y)$ in technology $T$ stated in the form (11). The following two axioms state that the volume vectors $X^{V}$ and $Y^{V}$ can be scaled by a factor $\alpha \geq 0$, provided the ratio measures change as stated in conditions (5)-(10). Because the cases of contraction $(0 \leq \alpha<1)$ and expansion $(\alpha>1)$ are sufficiently different, we consider them separately.

Axiom 4 (Selective proportional contraction). Let $(X, Y) \in T$. Then for all $0 \leq \alpha<1$,

$$
\left(\begin{array}{c}
X^{\alpha} \\
Y^{\alpha}
\end{array}\right)=\left(\begin{array}{c}
\alpha X^{V}, X^{F}, \alpha X^{D}+(1-\alpha) \bar{p}, X^{U}, \alpha X^{P} \\
\alpha Y^{V}, Y^{F}, \alpha Y^{D}+(1-\alpha) \bar{q}, \alpha Y^{P}
\end{array}\right) \in T
$$

Consider the expansion scenario with $\alpha>1$. As discussed in $\S 4.6, \alpha$ can increase while all proportional and upward-proportional ratio inputs are within the bounds (2), after which $\alpha$ cannot increase any further. In contrast, if the upper bounds on proportional ratio outputs are reached, $\alpha$ can increase further but such ratio outputs remain constant at their maximum level.

For any two vectors $a$ and $b$ of the same dimension $K$, let $\overline{\min }\{a, b\}$ be the vector of dimension $K$ whose components are equal to the minimum of the corresponding components of $a$ and $b: \overline{\min }\{a, b\}_{k}=\min \left\{a_{k}, b_{k}\right\}$, for all $k=1, \ldots, K$.

Axiom 5 (Selective proportional expansion). Let $(X, Y) \in T$. Then for all $\alpha>1$ such that $\alpha X^{U} \leq \bar{X}^{U}$ and $\alpha X^{P} \leq \bar{X}^{P}$

$$
\left(\begin{array}{c}
X^{\alpha} \\
Y^{\alpha}
\end{array}\right)=\left(\begin{array}{c}
\alpha X^{V}, X^{F}, X^{D}, \alpha X^{U}, \alpha X^{P} \\
\alpha Y^{V}, Y^{F}, Y^{D}, \min \left\{\alpha Y^{P}, \bar{Y}^{P}\right\}
\end{array}\right) \in T
$$


We need two further assumptions for the development of R-CRS technology with ratio measures.

Data Assumption 3. Vectors $\bar{p}$ and $\bar{q}$ are within the bounds stated in (2): $\bar{p} \leq \bar{X}^{D}$ and $\bar{q} \leq \bar{Y}^{D}$. Furthermore, $X_{j}^{D} \leq \bar{p}$ and $Y_{j}^{D} \geq \bar{q}$, for all $j \in J$.

Data Assumption 4. One or more of the following three conditions is satisfied:

(a) $\mathrm{I}^{V} \neq \varnothing$ and $X_{j}^{V} \neq \mathbf{0}$, for all $j \in J$;

(b) $\mathrm{I}^{U} \neq \varnothing$ and $X_{j}^{U} \neq \mathbf{0}$, for all $j \in J$;

(c) $\mathrm{I}^{P} \neq \varnothing$ and $X_{j}^{P} \neq \mathbf{0}$, for all $j \in J$.

Note that Data Assumption 4 makes Data Assumption 1 redundant. ${ }^{15}$

\subsection{Definition of the R-CRS technology}

The following definition introduces the R-CRS technology.

Definition 2. The R-CRS technology $T_{\mathrm{CRS}}^{\mathrm{R}}$ is the intersection of all technologies (sets) $T \subset \mathbb{R}_{+}^{m} \times \mathbb{R}_{+}^{s}$ that satisfy Axioms $1-5 .{ }^{16}$

Theorem 2. Let Data Assumptions 2-4 be true. Technology $T_{\mathrm{CRS}}^{\mathrm{R}}$ is the set of all DMUs $(X, Y) \in \mathbb{R}_{+}^{m} \times \mathbb{R}_{+}^{s}$ that satisfy (2) and for which there exist vectors $\lambda, \alpha, \beta \in \mathbb{R}^{n}$ such that

$$
\begin{aligned}
& \sum_{j \in J} \lambda_{j} \beta_{j} \alpha_{j} Y_{j}^{V} \geq Y^{V}, \\
& \sum_{j \in J} \lambda_{j} \beta_{j} \alpha_{j} X_{j}^{V} \leq X^{V}, \\
& \forall j \in J, \text { if } \lambda_{j}>0 \text { then } \\
& \quad\left(Y_{j}^{F} \geq Y^{F},\right. \\
& X_{j}^{F} \leq X^{F}, \\
& \quad \alpha_{j} Y_{j}^{D}+\left(1-\alpha_{j}\right) \bar{q} \geq Y^{D}, \\
& \quad \alpha_{j} X_{j}^{D}+\left(1-\alpha_{j}\right) \bar{p} \leq X^{D}, \\
& \quad \beta_{j} X_{j}^{U} \leq X^{U}, \\
& \beta_{j} \alpha_{j} Y_{j}^{P} \geq Y^{P}, \\
& \left.\quad \beta_{j} \alpha_{j} X_{j}^{P} \leq X^{P}\right), \\
& \mathbf{1}^{\top} \lambda=1 \\
& \alpha \leq \mathbf{1} \\
& \beta \geq \mathbf{1} \\
& \lambda, \alpha, \beta \geq \mathbf{0} .
\end{aligned}
$$

Conditions (12) are intuitively clear. Consider any observed $\operatorname{DMU}\left(X_{j}, Y_{j}\right), j \in J$, for which $\lambda_{j}>0$. Such a DMU is contracted by the scaling factor $\alpha_{j} \leq 1$ and further expanded by the factor $\beta_{j} \geq 1 .{ }^{17}$ Each condition (12.3)-(12.9) represents the response of

\footnotetext{
${ }^{15}$ Also note that Data Assumption 4 does not require that the technology has volume measures (although this is required for the properties of R-CRS models established in §6.2.) This makes our development applicable to DEA models where all inputs and outputs are ratios. As pointed in Example 4, models of this type are common in the assessment of socio-economic performance of countries.

${ }^{16} \mathrm{It}$ is straightforward to prove that technology $T_{\mathrm{CRS}}^{\mathrm{R}}$ satisfies Axioms $1-5$ and bounds (2).

${ }^{17}$ Note that the factor $\beta_{j}$ corresponds to $\alpha$ in Axiom 5. We use notation $\beta_{j}$ for a better differentiation between the contraction and expansion cases in the statement of Theorem 2 .
} 


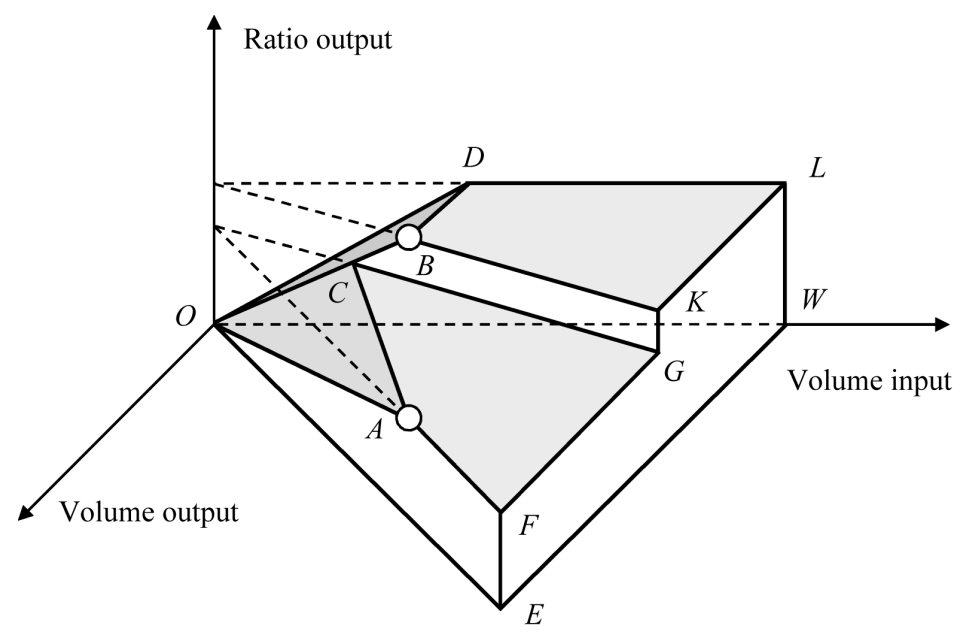

Figure 5: Downward-proportional ratio output in technology $T_{\mathrm{CRS}}^{\mathrm{R}}$.

a particular type of ratio input or output to the scaling with $\alpha_{j}$ and $\beta_{j}$ discussed in $\S 4$ and stated by Axioms 4 and 5 . For each $j$, denote the resulting $\operatorname{DMU}\left(\tilde{X}_{j}, \tilde{Y}_{j}\right)$. (To avoid excessive technicalities, we assume that all DMUs $\left(\tilde{X}_{j}, \tilde{Y}_{j}\right)$ are within the bounds (2). A full explanation without this simplifying assumption is given in the proof of Theorem 2.) Similar to the case of R-VRS, the signs of inequalities (12.3)-(12.9) imply that the scaled observed DMUs $\left(\tilde{X}_{j}, \tilde{Y}_{j}\right)$ that are used in the convex combinations of volume measures in (12.1) and (12.2) with a positive $\lambda_{j}$ are not worse on all ratio measures than the $\operatorname{DMU}(X, Y)$.

If Data Assumptions 2-4 are true, the following two statements hold.

Proposition 2. Technology $T_{\mathrm{CRS}}^{\mathrm{R}}$ is a closed set.

Proposition 3. $T_{\mathrm{VRS}}^{\mathrm{R}} \subset T_{\mathrm{CRS}}^{\mathrm{R}} \cdot{ }^{18}$

Figure 5 shows an example of technology $T_{\mathrm{CRS}}^{\mathrm{R}}$ induced by DMUs $A$ and $B$, and incorporates a downward-proportional ratio output. The latter is scaled down to zero together with the volume measures but remains constant if the volume measures are increased from the observed levels.

The technology in Figure 6 is induced by a single DMU $A$ and incorporates a downwardproportional ratio input. This technology includes the DMUs above the facets $A B C$ and $A C D E$, and is unbounded with respect to its volume and ratio inputs. Because this technology is induced by a single DMU, it is convex - this is no longer true in the case of two or more observed DMUs.

\subsection{Linearized statement of the $R$-CRS technology}

The statement by Theorem 2 is intuitively clear but not sufficiently operational. Theorem 3 below restates the conditions for technology $T_{\mathrm{CRS}}^{\mathrm{R}}$ in a simpler but equivalent form which has obvious computational advantages. The main idea of this transformation is based on the substitution of variables in Theorem 2 by $\mu_{j}=\lambda_{j} \alpha_{j}\left(\beta_{j}-1\right)$ and $\nu_{j}=\lambda_{j}\left(1-\alpha_{j}\right)$, for all $j \in J$.

\footnotetext{
${ }^{18} \mathrm{We}$ assume that ratio inputs and outputs of all types are referred to the sets $\mathrm{I}^{R}$ and $\mathrm{O}^{R}$ of technology $T_{\mathrm{VRS}}^{\mathrm{R}}$.
} 
Theorem 3. Let Data Assumptions 2-4 be true. Technology $T_{\mathrm{CRS}}^{\mathrm{R}}$ is the set of all DMUs $(X, Y) \in \mathbb{R}_{+}^{m} \times \mathbb{R}_{+}^{s}$ that satisfy (2) and for which there exist vectors $\lambda, \mu, \nu \in \mathbb{R}^{n}$ such that the following conditions hold.

Conditions for volume outputs and inputs:

$\sum_{j \in J}\left(\lambda_{j}+\mu_{j}-\nu_{j}\right) Y_{j}^{V} \geq Y^{V}$,
$\sum_{j \in J}\left(\lambda_{j}+\mu_{j}-\nu_{j}\right) X_{j}^{V} \leq X^{V}$,

Conditions for fixed ratio outputs and inputs:

$$
\begin{array}{ll}
\left(\lambda_{j}+\mu_{j}\right)\left(Y_{j}^{F}-Y^{F}\right) \geq \mathbf{0}, & \forall j \in J, \\
\left(\lambda_{j}+\mu_{j}\right)\left(X_{j}^{F}-X^{F}\right) \leq \mathbf{0}, & \forall j \in J,
\end{array}
$$

Conditions for downward-proportional ratio outputs:

$$
\begin{array}{ll}
\left(\lambda_{j}-\nu_{j}\right) Y_{j}^{D}+\nu_{j} \bar{q} \geq \lambda_{j} Y^{D}, & \forall j \in J, \\
\mu_{j}\left(Y_{j}^{D}-Y^{D}\right) \geq \mathbf{0}, & \forall j \in J,
\end{array}
$$

Conditions for downward-proportional ratio inputs:

$$
\begin{array}{ll}
\left(\lambda_{j}-\nu_{j}\right) X_{j}^{D}+\nu_{j} \bar{p} \leq \lambda_{j} X^{D}, & \forall j \in J, \\
\mu_{j}\left(X_{j}^{D}-X^{D}\right) \leq \mathbf{0}, & \forall j \in J,
\end{array}
$$

Conditions for upward-proportional ratio inputs:

$$
\begin{array}{ll}
\left(\lambda_{j}+\mu_{j}-\nu_{j}\right) X_{j}^{U} \leq\left(\lambda_{j}-\nu_{j}\right) X^{U}, & \forall j \in J, \\
\lambda_{j}\left(X_{j}^{U}-X^{U}\right) \leq \mathbf{0}, & \forall j \in J,
\end{array}
$$

Conditions for proportional ratio outputs:

$$
\begin{array}{ll}
\left(\lambda_{j}+\mu_{j}-\nu_{j}\right) Y_{j}^{P} \geq \lambda_{j} Y^{P}, & \forall j \in J, \\
\text { if }\left(\mu_{j}>0 \text { and } Y_{r}^{P}>0\right) \text { then } Y_{j r}^{P}>0, \forall r \in \mathrm{O}^{P}, & \forall j \in J,
\end{array}
$$

Conditions for proportional ratio inputs:

$$
\left(\lambda_{j}+\mu_{j}-\nu_{j}\right) X_{j}^{P} \leq \lambda_{j} X^{P}, \quad \forall j \in J,
$$

General conditions:

$$
\begin{aligned}
& \mathbf{1}^{\top} \lambda=1, \\
& \lambda_{j}-\nu_{j} \geq 0,
\end{aligned}
$$$$
\forall j \in J
$$

The statement of technology $T_{\mathrm{CRS}}^{\mathrm{R}}$ in Theorem 3 is less intuitive than its equivalent statement in Theorem 2. However, its conditions (13) are computationally simpler than conditions (12). Three remarks are worth making regarding the above results.

1. Each of the conditions (12.5)-(12.8) in Theorem 2 corresponds to a pair of conditions in Theorem 3. For example, the conditional inequality (12.5) (required to be true if $\left.\lambda_{j}>0\right)$ corresponds to two unconditional inequalities (13.5a) and (13.5b).

2. If some types of ratios are not used, the corresponding conditions are removed from the statements of Theorems 2 and 3. For example, if $\mathrm{I}^{D}=\mathrm{O}^{P}=\varnothing$, conditions (12.6) and (12.8) are removed from Theorem 2, and (13.6a), (13.6b), (13.8a) and (13.8b) are removed from Theorem 3 .

In particular, if there are no ratio measures, technology $T_{\mathrm{CRS}}^{\mathrm{R}}$ becomes the conventional CRS technology. Indeed, in this case conditions (12.3)-(12.9) are omitted from (12). 


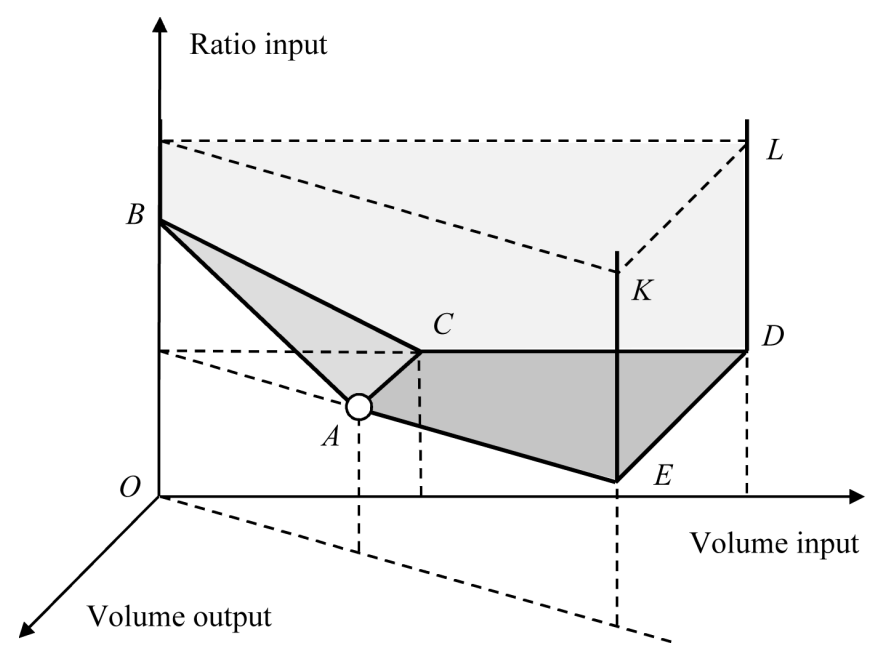

Figure 6: Downward-proportional ratio input in technology $T_{\mathrm{CRS}}^{\mathrm{R}}$.

By denoting $\hat{\lambda}_{j}=\lambda_{j} \beta_{j} \alpha_{j}$ and removing conditions (12.10)-(12.12) as redundant, we obtain the standard CRS technology stated in terms of vector $\hat{\lambda}$. The same result follows from conditions (13). In this case conditions (13.3)-(13.9) are omitted. The standard CRS technology is obtained by denoting $\tilde{\lambda}_{j}=\lambda_{j}+\mu_{j}-\nu_{j}$ and removing conditions (13.10) and (13.11) as redundant.

3. If all components of vectors $Y_{j}^{P}$ are strictly positive for all $j \in J$, then condition $(13.8 \mathrm{~b})$ is obviously redundant and can be omitted.

\subsection{Special case: the R-CRS technology with fixed ratio measures}

Suppose that technology $T_{\mathrm{CRS}}^{\mathrm{R}}$ includes both volume inputs and outputs, and ratio measures of the fixed type only. Denote such a technology $T_{\mathrm{CRS}}^{\mathrm{F}}$. It is described by Theorem 2 in which conditions (12.5)-(12.9) are omitted, and by Theorem 3 in which conditions (13.5a)(13.9) are removed.

An alternative, and simpler, statement of the same technology $T_{\mathrm{CRS}}^{\mathrm{F}}$ is given by the following proposition, under the additional assumption $Y^{V} \neq \mathbf{0}$ which should not be problematic in practical applications. An advantage of this statement is that it utilizes a single vector $\lambda$.

Proposition 4. Let Data Assumptions 2-4 be true. Consider any $(X, Y) \in \mathbb{R}_{+}^{m} \times \mathbb{R}_{+}^{s}$ such that $Y^{V} \neq \mathbf{0}$. Then $(X, Y) \in T_{\mathrm{CRS}}^{\mathrm{F}}$ if and only if $(X, Y)$ satisfies $(2)$ and there exists a vector $\lambda \in \mathbb{R}^{n}$ such that conditions (4.1)-(4.4) and (4.6) are true.

Technology $T_{\mathrm{CRS}}^{\mathrm{F}}$ models an important practical scenario in which volume measures satisfy the assumption of CRS and can be scaled up and down (because the normalizing condition (4.5) is missing in the above statement), while the ratio measures control for the environment and quality, and remain unchanged. Technology $T_{\mathrm{CRS}}^{\mathrm{F}}$ can be regarded as $a$ partial cone extension to technology $T_{\mathrm{VRS}}^{\mathrm{R}}$, of which the technology of Ruggiero (1996) is a special case.

Figure 7 shows technology $T_{\mathrm{CRS}}^{\mathrm{F}}$ induced by two observed DMUs $A$ and $B$. It has a fixed ratio output and its section $E F G K L W$ is not convex. As the volume input and output are scaled up or down, the ratio output remains constant. 


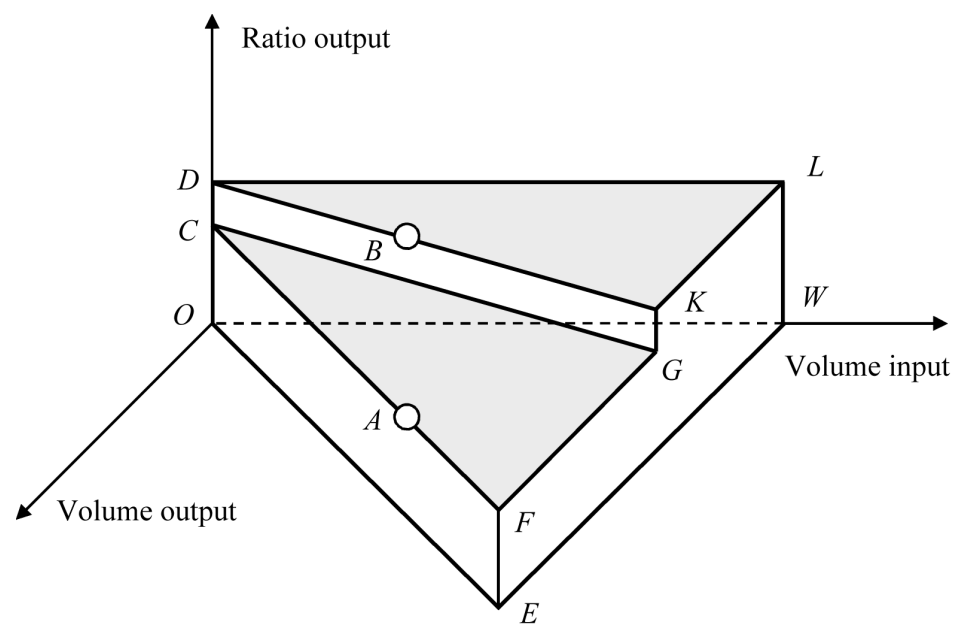

Figure 7: Fixed ratio output in technology $T_{\mathrm{CRS}}^{\mathrm{F}}$.

\section{DEA models with ratio measures}

The formulation of R-VRS and R-CRS DEA models based on technologies $T_{\mathrm{VRS}}^{\mathrm{R}}$ and $T_{\mathrm{CRS}}^{\mathrm{R}}$ is straightforward. For simplicity, below we limit our discussion to models with radial input and output efficiency measures. These are easy to extend to measures based on the general directional distance function (Chambers et al., 1998), but this leads to lengthier formulations and is not pursued further.

\subsection{Formulation}

First, consider the case of input minimization. In production theory, the input radial efficiency of $\operatorname{DMU}\left(X_{o}, Y_{o}\right)$ is usually defined by attaching the improvement factor $\theta$ only to its volume inputs, while using ratio measures to control for environment and quality of production. However, in the broader context, we may also be interested in possible improvements to ratio measures. ${ }^{19}$ In such cases $\theta$ may not have the conventional meaning of input radial efficiency, but it is still a measure of input improvement. For simplicity, we refer to input radial efficiency as a unifying term applicable to both volume and ratio measures.

As a general case, suppose it is desirable to measure the input radial efficiency of DMU $\left(X_{o}, Y_{o}\right)$ only with respect to a subset of inputs (for example, only volume inputs). To state this, we attach the improvement factor $\theta$ to a non-empty subset of inputs $\mathrm{I}^{\theta} \subseteq \mathrm{I}$ and define the improved vector $X_{o}(\theta)$ as follows:

$$
X_{o i}(\theta)= \begin{cases}\theta X_{o i}, & \text { if } i \in \mathrm{I}^{\theta}, \\ X_{o i}, & \text { if } i \in \mathrm{I} \backslash \mathrm{I}^{\theta} .\end{cases}
$$

\footnotetext{
${ }^{19}$ As an illustration, the DM in Example 1 may ask 1) what is the best practice (lowest) mortality rate $\mathcal{R}_{4}$ that an observed hospital should aim at without changing its other volume and ratio inputs and outputs, or 2 ) what is the lowest contextual proportion of vaccinated people $\mathcal{R}_{8}$ that should not affect the performance of a hospital? In both cases the improvement factor $\theta$ is attached to the corresponding ratio input. As another illustration, the efficiency of countries in the context of Example 4 is also measured with respect to ratio measures.
} 
Based on Theorem 1, the input radial efficiency $E_{\mathrm{VRS}}^{1}\left(X_{o}, Y_{o}\right)$ of the $\mathrm{DMU}\left(X_{o}, Y_{o}\right) \in T_{\mathrm{VRS}}^{\mathrm{R}}$ is equal to the optimal value $\theta^{*}$ in the following model: ${ }^{20}$

$\left[\mathrm{M}_{\text {VRS }}^{1}\right]$

$\theta^{*}=\min \theta$,

subject to: $(X, Y)=\left(X_{o}(\theta), Y_{o}\right)$ satisfies $(2)$ and $(4), \theta$ sign free.

Similarly, to allow the assessment of the output radial efficiency of DMU $\left(X_{o}, Y_{o}\right)$ with respect to a non-empty subset of outputs $\mathrm{O}^{\eta} \subseteq \mathrm{O}$, define

$$
Y_{o r}(\eta)= \begin{cases}\eta Y_{o r}, & \text { if } r \in \mathrm{O}^{\eta}, \\ Y_{o r}, & \text { if } r \in \mathrm{O} \backslash \mathrm{O}^{\eta} .\end{cases}
$$

The output radial efficiency $E_{\mathrm{VRS}}^{2}\left(X_{o}, Y_{o}\right)$ of the DMU $\left(X_{o}, Y_{o}\right) \in T_{\mathrm{VRS}}^{\mathrm{R}}$ is obtained by inverting the maximum output-improvement factor $\eta^{*}$ in the model

$\left[\mathbb{M}_{\mathrm{VRS}}^{2}\right]$

$$
\eta^{*}=\max \eta
$$

subject to: $(X, Y)=\left(X_{o}, Y_{o}(\eta)\right)$ satisfies $(2)$ and $(4), \eta$ sign free.

In the case of CRS, we replace conditions (4) in models $\mathbb{M}_{\text {VRS }}^{1}$ and $\mathbb{M}_{\text {VRS }}^{2}$ by conditions (13), and denote the resulting R-CRS models $\mathbb{M}_{\text {CRS }}^{1}$ and $\mathbb{M}_{\text {CRS }}^{2}$, respectively. For any $\operatorname{DMU}\left(X_{o}, Y_{o}\right) \in T_{\mathrm{CRS}}^{\mathrm{R}}$, its input radial efficiency $E_{\mathrm{CRS}}^{1}\left(X_{o}, Y_{o}\right)$ is equal to the optimal value $\theta^{*}$ in model $\mathbb{M}_{\mathrm{CRS}}^{1}$. Its output-radial efficiency $E_{\mathrm{CRS}}^{2}\left(X_{o}, Y_{o}\right)$ is reciprocal to the optimal value $\eta^{*}$ in model $\mathbb{M}_{\mathrm{CRS}}^{2}$.

\subsection{Properties of DEA models with ratio measures}

Below we obtain some basic properties of the R-VRS and R-CRS DEA models based on technologies with ratio data, and the corresponding efficiency measures. Because these models are based on Theorems 1-3, the relevant Data Assumptions 1-4, as specified in the above theorems, are assumed to be true. We also require an additional assumption concerning the nonnegativity of data.

Data Assumption 5. $\mathrm{I}^{V} \neq \varnothing, \mathrm{O}^{V} \neq \varnothing$, and all volume inputs and volume outputs of all observed DMUs $j \in J$ and the DMU $\left(X_{o}, Y_{o}\right)$ are strictly positive. Furthermore, the set $\mathrm{I}^{V} \cap \mathrm{I}^{\theta} \neq \varnothing$ in models $\mathbb{M}_{\mathrm{VRS}}^{1}$ and $\mathbb{M}_{\mathrm{CRS}}^{1}$, and $\mathrm{O}^{V} \cap \mathrm{O}^{\eta} \neq \varnothing$ in models $\mathbb{M}_{\mathrm{VRS}}^{2}$ and $\mathbb{M}_{\mathrm{CRS}}^{2}{ }^{21}$

Proposition 5. The optimum values in models $\mathbb{M}_{\mathrm{VRS}}^{1}, \mathbb{M}_{\mathrm{VRS}}^{2}, \mathbb{M}_{\mathrm{CRS}}^{1}$, and $\mathbb{M}_{\mathrm{CRS}}^{2}$ are attained. Furthermore,

$$
0<E_{\mathrm{CRS}}^{i}\left(X_{o}, Y_{o}\right) \leq E_{\mathrm{VRS}}^{i}\left(X_{o}, Y_{o}\right) \leq 1, \quad i=1,2 .
$$

We now refer to the known property of conventional CRS DEA models, namely, that the input and output radial efficiencies of any DMU are always equal. Because $T_{\mathrm{CRS}}^{\mathrm{R}}$ is generally not a cone technology, the above property does not automatically extend to the models based on it. The next statement identifies conditions under which the two efficiency measures remain equal.

Proposition 6. In addition to Data Assumptions 2-5, let

(a) $\mathrm{I}^{\theta}=\mathrm{I}^{\mathrm{V}} \cup \mathrm{I}^{\mathrm{P}}, \mathrm{O}^{\eta}=\mathrm{O}^{\mathrm{V}} \cup \mathrm{O}^{\mathrm{P}}$,

(b) $\mathrm{I}^{\mathrm{D}}=\mathrm{O}^{\mathrm{D}}=\mathrm{I}^{\mathrm{U}}=\varnothing$,

(c) if $\mathrm{O}^{\mathrm{P}} \neq \varnothing$ then $\bar{Y}_{r}^{P}=+\infty, \forall r \in \mathrm{O}^{\mathrm{P}}$.

Then $E_{\mathrm{CRS}}^{1}\left(X_{o}, Y_{o}\right)=E_{\mathrm{CRS}}^{2}\left(X_{o}, Y_{o}\right)$.

\footnotetext{
${ }^{20}$ According to Proposition 5, the objective functions of all models in this section are attained.

${ }^{21}$ In the Appendix we replace Data Assumption 5 by a significantly weaker but more technical assumption that is actually needed for the proofs of Propositions $5-7$.
} 
As an example, consider technology $T_{\mathrm{CRS}}^{\mathrm{F}}$ stated in Proposition 4, in which all inputs and outputs are either volume or fixed ratio measures. By Proposition 6, the input radial efficiency of any DMU $\left(X_{o}, Y_{o}\right) \in T_{\mathrm{CRS}}^{\mathrm{F}}$ measured with respect to all volume inputs is equal to its output radial efficiency measured with respect to all volume outputs.

Proposition 7. (a) In models $\mathbb{M}_{\mathrm{VRS}}^{1}, \mathbb{M}_{\mathrm{VRS}}^{2}$ and $\mathbb{M}_{\mathrm{CRS}}^{1}$, all bounds (2) are redundant and can be removed from the formulation.

(b) In model $\mathbb{M}_{\mathrm{CRS}}^{2}$, all bounds (2) are redundant and can be removed from the formulation, except the bounds on the ratio outputs $r \in \mathrm{O}^{P} \cap \mathrm{O}^{\eta}$.

According to Proposition 7 , in model $\mathbb{M}_{\text {CRS }}^{2}$ we need to specify the bounds only for those proportional ratio outputs that have the improvement factor $\eta$ attached to them. Such bounds take on the form $\eta Y_{\text {or }}^{P} \leq \bar{Y}_{r}^{P}, \forall r \in \mathrm{O}^{P} \cap \mathrm{O}^{\eta}$.

\subsection{Dual formulations}

Below we construct the dual multiplier forms of the R-VRS models in the practically important special case when the radial improvement factor is attached only to the volume inputs or outputs. A similar dual formulation can be given for the R-CRS models if all ratio inputs and outputs are of the fixed type only.

To be specific, consider the input-minimization model $\mathbb{M}_{\mathrm{VRS}}^{1}$ in which all volume inputs are minimized: $\mathrm{I}^{\theta}=\mathrm{I}^{V}$. To simplify the exposition, we also assume that no finite upper bounds (2) are specified. (By Proposition 7, such bounds are redundant in the R-VRS models). In this case model $\mathbb{M}_{\text {VRS }}^{1}$ is a linear program that minimizes $\theta$ over the feasible set described by linear inequalities (4), in which the DMU $\left(X^{V}, X^{R}, Y^{V}, Y^{R}\right)$ is substituted by $\left(\theta X_{o}^{V}, X_{o}^{R}, Y_{o}^{V}, Y_{o}^{R}\right)$.

Define the subset of observed DMUs $J_{o} \subseteq J$ as follows: $J_{o}=\left\{j \in J \mid X_{o}^{R} \geq X_{j}^{R}, Y_{o}^{R} \leq\right.$ $Y_{j}^{R}$ \}. The DMUs $j \in J_{o}$ have lower or equal levels of ratio inputs, and higher or equal levels of ratio outputs, compared to DMU $\left(X_{o}, Y_{o}\right)$ under the assessment. If the ratio inputs represent the quality of the environment in which the DMUs operate, and the ratio outputs represent the quality of products or services, then the observed DMUs in the set $J_{o}$ operate in the environment of the same or lower quality compared to DMU $\left(X_{o}, Y_{o}\right)$, and the quality of their products and services is not lower than of the latter DMU. ${ }^{22}$

Proposition 8. Let $\mathrm{I}^{\theta}=\mathrm{I}^{V}$. Then the dual to model $\mathbb{M}_{\mathrm{VRS}}^{1}$ can be stated as

$$
\begin{aligned}
& \max u^{\top} Y_{o}^{V}+w, \\
& \text { subject to } \\
& v^{\top} X_{o}^{V}=1, \\
& u^{\top} Y_{j}^{V}-v^{\top} X_{j}^{V}+w \leq 0, \quad \forall j \in J_{o}, \\
& u, v \geq \mathbf{0}, w \text { sign free, }
\end{aligned}
$$

where the variable vectors $u$ and $v$ are of dimensions $\left|\mathrm{O}^{V}\right|$ and $\left|\mathrm{I}^{V}\right|$, respectively, and $w$ is a scalar variable.

\footnotetext{
${ }^{22}$ If $\left(X_{o}, Y_{o}\right)$ is an observed DMU $j_{o}$ then $j_{o} \in J_{o}$ and $J_{o} \neq \varnothing$. If DMU $\left(X_{o}, Y_{o}\right)$ is not observed, the set $J_{o}$ may be empty. In this case conditions (4) (more precisely, conditions (4.3)-(4.6)) cannot be satisfied. This means that DMU $\left(X_{o}, Y_{o}\right)$ is not in technology $T_{\mathrm{VRS}}^{\mathrm{R}}$. In this case model $\mathbb{M}_{\mathrm{VRS}}^{1}$ is infeasible, and its dual model (14) has an unbounded optimal value.
} 
Program (14) is "almost" the standard multiplier model except for it limits the number of comparator DMUs to the set $J_{o}$, which generally depends on DMU $\left(X_{o}, Y_{o}\right)$. This is equivalent to the assessment of efficiency in the standard VRS technology (with volume inputs and outputs only) induced by the restricted set of observed DMUs $J_{o}{ }^{23}$

Model (14) allows the standard interpretation associated with conventional VRS multiplier models. In particular, it retains the meaning of the optimal weights $u$ and $v$ as the shadow prices of outputs and shadow costs of inputs, respectively, and the free variable $w$ can be used to identify scale properties of the DMUs. As noted, the only difference with the conventional case is that each DMU $\left(X_{o}, Y_{o}\right)$ is compared with the qualifying DMUs $j \in J_{o}$, and the other DMUs from the set $J \backslash J_{o}$ are disallowed as potential comparators.

The dual to the output-maximization R-VRS model $\mathbb{M}_{\text {VRS }}^{2}$ is obtained in a similar way, assuming that $\mathrm{O}^{\eta}=\mathrm{O}^{V}$.

A similar treatment is applicable to the R-CRS models $\mathbb{M}_{\text {CRS }}^{1}$ and $\mathbb{M}_{\text {CRS }}^{2}$, provided the ratio inputs and outputs are of the fixed type only and, as above, the improvement factors are attached only to the volume measures. To be specific, consider the input-minimization model $\mathbb{M}_{\text {CRS }}^{1}$ with fixed ratio inputs and outputs. According to Proposition 4, the underlying technology $T_{\mathrm{CRS}}^{\mathrm{F}}$ is described by inequalities (4) from which the normalizing equality (4.5) is removed. Repeating the proof of Proposition 8, it is straightforward to verify that the dual to model $\mathbb{M}_{\text {CRS }}^{1}$ (under the specified conditions) can be stated as program (14) in which the scalar variable $w$ is omitted.

The investigation of the dual programs to models $\mathbb{M}_{\text {CRS }}^{1}$ and $\mathbb{M}_{\text {CRS }}^{2}$ that incorporate ratio measures other than of the fixed type goes beyond the scope of this paper.

\section{Computational issues and example}

Below we consider computational approaches to the R-VRS and R-CRS models and provide a computational example.

\subsection{Computational approaches}

To be specific, we consider the solution of input-minimization R-VRS and R-CRS models $\mathbb{M}_{\text {VRS }}^{1}$ and $\mathbb{M}_{\text {CRS }}^{1}$ based on conditions (4) and (13), respectively. The case of outputmaximization models is similar, with a minor difference in the treatment of bounds (2) as stated in Proposition 7.

Depending on which inputs are included in the set $\mathrm{I}^{\theta}$ and (in the case of R-CRS) what types of ratio measures are used, models $\mathbb{M}_{\text {VRS }}^{1}$ and $\mathbb{M}_{\text {CRS }}^{1}$ can take on linear, mixed integer linear and nonlinear forms. Below we briefly discuss these three cases.

1. Linear programming. This is the simplest case that arises if the radial improvement factor $\theta$ is attached only to volume inputs of the DMU $\left(X_{o}, Y_{o}\right)$. Then the R-VRS model $\mathbb{M}_{\text {VRS }}^{1}$ is a linear program with respect to $\lambda$ and $\theta$. The R-CRS model $\mathbb{M}_{\text {CRS }}^{1}$ is also a linear program with respect to $\lambda, \mu, \nu$ and $\theta$, if condition (13.8b) is not used.

\footnotetext{
${ }^{23}$ It is interesting to note that the envelopment R-VRS model $\mathbb{M}_{\text {VRS }}^{1}$ based on conditions (4) can also be restated using the set $J_{o}$. Indeed, for any $j \in J_{o}$ the corresponding inequalities (4.3) and (4.4) are trivially true and can be omitted as redundant. Furthermore, from (4.3) and (4.4) we have $\lambda_{j}=0$, for all $j \in J \backslash J_{o}$. Therefore, conditions (4) are equivalently restated by omitting both groups of inequalities (4.3) and (4.4), and by performing the summation in (4.1) and (4.2) over the set $J_{o}$ instead of $J$. Model $\mathbb{M}_{\text {VRS }}^{1}$ based on the resulting restatement of conditions (4) is the standard dual of the multiplier model (14).
} 
Table 2: Hospital data.

\begin{tabular}{cccc}
\hline Hospital & Costs $(£)$ & Patients & Target time $(\%)$ \\
\hline$A$ & 40,000 & 70 & 85 \\
$B$ & 30,000 & 50 & 55 \\
$C$ & 60,000 & 120 & 90 \\
$D$ & 25,000 & 65 & 20 \\
\hline
\end{tabular}

2. Mixed integer linear programming. This is the general case of the R-VRS model $\mathbb{M}_{\mathrm{VRS}}^{1}$ in which the improvement factor $\theta$ may be attached to any inputs. For the R-CRS model $\mathbb{M}_{\text {CRS }}^{1}$ this case is restricted to the situation in which $\theta$ is attached to any volume inputs or ratio inputs of the fixed type only. In this case, the nonlinear inequalities in either model can be linearized by the well-known method of treatment of "either-or" conditions. This transforms models $\mathbb{M}_{\mathrm{VRS}}^{1}$ and $\mathbb{M}_{\mathrm{CRS}}^{1}$ to mixed integer linear programs. ${ }^{24}$

3. Nonlinear programming. This is the general case of the R-CRS model $\mathbb{M}_{\text {CRS }}^{1}$ in which the factor $\theta$ may be attached to any inputs. Note that the corresponding inequalities become nonlinear. For example, (13.6a) contains the product $\theta \lambda_{j}$ on its right-hand side. In this case a simple line search procedure can be programmed that requires solving a (mixed integer) linear program at each step. The idea is to identify the value $\theta^{*}$ such that the model $\mathbb{M}_{\text {CRS }}^{1}$ is feasible with $\theta^{*}$ and infeasible with $\theta=\theta^{*}-\varepsilon$, where $\varepsilon>0$ is the required precision. Then $\theta^{*}$ may be taken as the input radial efficiency of the $\operatorname{DMU}\left(X_{o}, Y_{o}\right)$ calculated with the required precision.

\subsection{A computational example}

To illustrate the use of new DEA models, consider the following variant of Example 1. Table 2 shows four hospitals: $A, B, C$, and $D$, their volume input (costs in British pounds), volume output (number of patients treated for a certain condition) and the proportion of patients treated within the target time (ratio $\mathcal{R}_{2}$ in Example 1). The latter is regarded as a ratio output.

To be specific, we assess the efficiency of the four hospitals with respect to the volume measures only. In particular, for the input radial efficiency we define $I^{\theta}=\{$ Costs $\}$, and for the output radial efficiency we define $\mathrm{O}^{\eta}=\{$ Patients $\}$. As above, we use notation $\mathbb{M}_{\text {VRS }}^{1}$ and $\mathbb{M}_{\text {VRS }}^{2}$ to denote the input-minimization and output-maximization R-VRS models, respectively. In the case of $\mathrm{R}$-CRS, we use models $\mathbb{M}_{\text {CRS }}^{1}$ and $\mathbb{M}_{\text {CRS }}^{2}$ based on the formulation of Theorem 3. According to Proposition 7, the upper bound on the ratio output (100\%) needs not specifying in any of these models.

We use this example to illustrate how the assumption of the ratio type can affect the resulting efficiency in the R-CRS model. Below we consider three scenarios in which the ratio output $\mathcal{R}_{2}$ is modeled as the fixed (F), downward-proportional (D) or proportional

\footnotetext{
${ }^{24}$ The treatment of condition (13.8b) in the R-CRS model $\mathbb{M}_{\mathrm{CRS}}^{1}$ requires an extra step. First, we introduce a new variable vector $\tau \in \mathbb{R}_{+}^{n}$ and note that condition (13.8b) can be restated as follows: if $\mu_{j}>0$, there exists a $\tau_{j} \geq 0$ such that $\tau_{j} Y_{j}^{P} \geq Y^{P}$. This is equivalent to the "either-or" statement: either $\mu_{j}=0$ or $\tau_{j} Y_{j}^{P} \geq Y^{P}, \forall j \in J$.
} 
(P) type. We add the corresponding letter to the model notation to indicate that the ratio output is treated as being of a particular type.

For example, $\mathbb{M}_{\text {CRS }}^{1}(\mathrm{D})$ is the input-minimization R-CRS model in which $\mathcal{R}_{2}$ is a downwardproportional ratio output defined by formula (7) with $q=0$. For hospital $A$ this model takes on the form: ${ }^{25}$

$$
\begin{aligned}
& \theta^{*}=\min \theta \\
& \text { subject to } \\
& 70\left(\lambda_{A}+\mu_{A}-\nu_{A}\right)+50\left(\lambda_{B}+\mu_{B}-\nu_{B}\right)+120\left(\lambda_{C}+\mu_{C}-\nu_{C}\right)+65\left(\lambda_{D}+\mu_{D}-\nu_{D}\right) \geq 70, \\
& 40\left(\lambda_{A}+\mu_{A}-\nu_{A}\right)+30\left(\lambda_{B}+\mu_{B}-\nu_{B}\right)+60\left(\lambda_{C}+\mu_{C}-\nu_{C}\right)+25\left(\lambda_{D}+\mu_{D}-\nu_{D}\right) \leq 40 \theta, \\
& 0.85\left(\lambda_{A}-\nu_{A}\right)+0 \nu_{A} \geq 0.85 \lambda_{A}, \\
& (0.85-0.85) \mu_{A} \geq 0, \\
& 0.55\left(\lambda_{B}-\nu_{B}\right)+0 \nu_{B} \geq 0.85 \lambda_{B}, \\
& (0.55-0.85) \mu_{B} \geq 0 \\
& 0.9\left(\lambda_{C}-\nu_{C}\right)+0 \nu_{C} \geq 0.85 \lambda_{C}, \\
& (0.9-0.85) \mu_{C} \geq 0, \\
& 0.2\left(\lambda_{D}-\nu_{D}\right)+0 \nu_{D} \geq 0.85 \lambda_{D}, \\
& (0.2-0.85) \mu_{D} \geq 0, \\
& \lambda_{A}+\lambda_{B}+\lambda_{C}+\lambda_{D}=1, \\
& \lambda_{A}-\nu_{A} \geq 0, \lambda_{B}-\nu_{B} \geq 0, \lambda_{C}-\nu_{C} \geq 0, \lambda_{D}-\nu_{D} \geq 0, \\
& \lambda_{A}, \lambda_{B}, \lambda_{C}, \lambda_{D} \geq 0, \mu_{A}, \mu_{B}, \mu_{C}, \mu_{D} \geq 0, \nu_{A}, \nu_{B}, \nu_{C}, \nu_{D} \geq 0, \theta \text { sign free. }
\end{aligned}
$$

Table 3 shows the efficiency of the four hospitals in different models. ${ }^{26}$ Comparing the efficiency of hospitals in the input-minimization R-CRS models, we observe that the least discriminating is model $\mathbb{M}_{\text {CRS }}^{1}(\mathrm{D})$. This is consistent with the fact that the assumption of the downward-proportional type, based on the worst-case scenario, is weaker than the assumptions of the fixed and proportional types. ${ }^{27} \mathrm{~A}$ similar observation can be made in the case of output maximization: model $\mathbb{M}_{\mathrm{CRS}}^{2}(\mathrm{D})$ is the least discriminating of the three $\mathrm{R}$-CRS models in this case.

Also note that the efficiency of each hospital is the same in the input-minimization RCRS model $\mathbb{M}_{\text {CRS }}^{1}(F)$ and the output-maximization R-CRS model $\mathbb{M}_{\text {CRS }}^{2}(F)$. This illustrates the statement of Proposition 6. Note that the same observation does not apply to models $\mathbb{M}_{\text {CRS }}^{1}(\mathrm{D})$ and $\mathbb{M}_{\text {CRS }}^{1}(\mathrm{P})$ : the efficiency of hospitals in these R-CRS models is generally different from their efficiency in the corresponding output maximization models $\mathbb{M}_{\mathrm{CRS}}^{2}(\mathrm{D})$ and $\mathbb{M}_{\text {CRS }}^{2}(\mathrm{P})$.

\section{The answer to the DM's dilemma in Example 1}

The DM's dilemma in Example 1 arises from the fact that none of the existing DEA models is suitable for the incorporation of ratio measures from $\mathcal{R}_{1}$ to $\mathcal{R}_{8}$. Their use would

\footnotetext{
${ }^{25}$ To be consistent with the description of the downward-proportional ratio output (7) and its use in program (13), we have transformed the target times shown in Table 2 as percentages, into fractions of 1 .

${ }^{26}$ All models were solved using a common commercial solver.

${ }^{27}$ The fixed type makes larger values of the ratio output (compared to the downward-proportional type) feasible for $\alpha<1$. The proportional type adds more points to the technology than the downwardproportional type for $\alpha>1$.
} 
Table 3: Efficiency in different models.

\begin{tabular}{ccccccccc}
\hline & \multicolumn{3}{c}{ Input-minimization models } & \multicolumn{4}{c}{ Output-maximization models } \\
\cline { 2 - 8 } Hospital & $\mathbb{M}_{\text {VRS }}^{1}$ & $\mathbb{M}_{\text {CRS }}^{1}(\mathrm{~F})$ & $\mathbb{M}_{\text {CRS }}^{1}(\mathrm{D})$ & $\mathbb{M}_{\text {CRS }}^{1}(\mathrm{P})$ & $\mathbb{M}_{\text {VRS }}^{2}$ & $\mathbb{M}_{\text {CRS }}^{2}(\mathrm{~F})$ & $\mathbb{M}_{\text {CRS }}^{2}(\mathrm{D})$ & $\mathbb{M}_{\text {CRS }}^{2}(\mathrm{P})$ \\
\hline$A$ & 1 & 0.875 & 1 & 1 & 1 & 0.875 & 1 & 1 \\
$B$ & 1 & 0.833 & 0.923 & 0.913 & 1 & 0.833 & 0.893 & 0.86 \\
$C$ & 1 & 1 & 1 & 0.952 & 1 & 1 & 1 & 0.93 \\
$D$ & 1 & 1 & 1 & 1 & 1 & 1 & 1 & 1 \\
\hline
\end{tabular}

result in an incorrect model of production technology and potentially lead to a flawed assessment of benchmark costs. The models developed in our paper are designed to resolve this type of problem.

The case of R-VRS is straightforward: the DM can use model $\mathbb{M}_{\mathrm{VRS}}^{1}$ that minimizes the costs (as in the above computational example). In particular, ratio outputs $\mathcal{R}_{1}$ and $\mathcal{R}_{2}$, and bad ratio inputs $\mathcal{R}_{6}$ and $\mathcal{R}_{7}$ (treated as outputs) should be incorporated in conditions (4.3). The ratio inputs $\mathcal{R}_{5}$ and $\mathcal{R}_{8}$, and bad ratio outputs $\mathcal{R}_{3}$ and $\mathcal{R}_{4}$ (viewed as inputs) are modeled by conditions (4.4).

In the case of R-CRS there is some modeling flexibility depending on the DM's willingness to make a specific assumption about the type of ratio measures. As a reasonable starting point, the DM may assume that $\mathcal{R}_{1}$ and $\mathcal{R}_{2}$ are downward-proportional ratio outputs, $\mathcal{R}_{3}$ and $\mathcal{R}_{4}$ are downward-proportional ratio inputs, and $\mathcal{R}_{5}$ is an upward-proportional ratio input. As discussed, this corresponds to the most conservative model of these ratios and expands the technology in the least possible way. Also, as argued in $\S 4$, the DM may model $\mathcal{R}_{6}$ as proportional ratio output, and $\mathcal{R}_{7}$ and $\mathcal{R}_{8}$ as fixed ratio output and input, respectively.

All of these ratios are incorporated in the cost-minimization R-CRS model $\mathbb{M}_{\text {CRS }}^{1}$ using the inequalities (13) of the corresponding type. For example, the downward-proportional success rate $\mathcal{R}_{1}$ is modeled by two inequalities (13.5a) and (13.5b). Although all ratio measures, except $\mathcal{R}_{5}$ and $\mathcal{R}_{6}$ are bounded above by 1 (or 100\%), by Proposition 7 , these do not need to be specified in the input-minimization model.

Assume the DM decides that the success rate $\mathcal{R}_{1}$, and readmission and mortality rates $\mathcal{R}_{3}$ and $\mathcal{R}_{4}$ do not significantly depend on the simultaneous reduction of costs and numbers of patients. In other words, scale is irrelevant for these measures. In this case $\mathcal{R}_{1}$ is modeled as a fixed ratio output by condition (13.3). The ratio inputs $\mathcal{R}_{3}$ and $\mathcal{R}_{4}$ are modeled as fixed ratio inputs in (13.4).

Furthermore, the DM may decide that the classification of the rate of radiotherapy treatments $\mathcal{R}_{5}$ as an upward-proportional input type is too conservative and wish to investigate the effect of reclassifying this ratio as the proportional input type. In this case $\mathcal{R}_{5}$ is incorporated in the model by conditions (13.8a) and (13.8b).

The computational example in $\S 7.2$ suggests that the reclassification of the above ratios to the fixed or proportional types should generally have a positive impact on the efficiency discrimination of the model. 


\section{Conclusion}

Various performance indicators in different industries and public organizations are available only in the form of ratios. A question as to how such data could be integrated in efficiency analysis has been debated for at least two decades. Despite a clear need of a definitive methodology, the existing literature has so far provided only a partial and often unconvincing answer as to how this could be done.

If ratio measures are used in conventional VRS and CRS DEA models, the underlying technology is generally modeled incorrectly: for example, it may include DMUs that cannot be produced. This implies that the efficient frontier becomes arbitrary and the efficiency cannot be reliably interpreted as a feasible improvement factor. This has a domino effect on such derived notions as scale and productivity change, both of which rely on a correct model of technology.

In our paper we address the above problem by developing new DEA models in which the production technology is modeled correctly, in accordance with the stated axioms. The latter explicitly account for different properties of volume and ratio measures. The new R-VRS and R-CRS technologies are formally derived from these axioms - this means that they are consistent with the stated assumptions and contain no arbitrary extensions to the observed set of DMUs.

The main result of our paper is the development of the R-VRS and R-CRS models of production technology suitable for ratio measures. Once the technologies are constructed, we can use different projection methods to obtain different DEA models and efficiency measures. An example of this is the assessment of radial input and output efficiency measures considered in $\S 6$, where the radial improvement factor can be applied to all, or only some, of the inputs or outputs. Other common efficiency measures can be used with the new technologies in a similar way. This includes measures based on directional distance functions, slack-based and hyperbolic measures of efficiency, to name a few common approaches. Properties of such models, related computational methods and methodological issues remain to be explored.

A number of other issues are left outside the scope of this paper. These include the statistical validation (testing) of assumptions, addressed for standard DEA models by Simar and Wilson (2002). Also unaddressed in this paper is the notion of scale efficiency. Its development for models with ratio data raises a number of conceptual problems and remains open for future research. 


\section{Appendix A. Proofs}

Proof of Theorem 1. The proof is a straightforward modification of the proof given by Podinovski (2005), and is omitted.

Proof of Proposition 1. This is similar to the proof Proposition 2 below and is omitted.

Proof of Theorem 2. Let $\tilde{T}_{\mathrm{CRS}}^{\mathrm{R}}$ be the set of all DMUs (11) that satisfy (2) and (12) with some vectors $\lambda, \alpha, \beta \in \mathbb{R}^{n}$. The proof of the theorem follows from Lemmas 1-3.

Lemma 1. Technology $\tilde{T}_{\mathrm{CRS}}^{\mathrm{R}}$ satisfies Axioms $1-3$.

Proof of Lemma 1. Axioms 1 and 2 are straightforward. Consider Axiom 3. Let DMUs $(\tilde{X}, \tilde{Y})$ and $(\hat{X}, \hat{Y})$ satisfy (12) with $\tilde{\lambda}, \tilde{\alpha}, \tilde{\beta}$ and $\hat{\lambda}, \hat{\alpha}, \hat{\beta}$, respectively, and let (3) be true. Let us prove that, for any $\gamma \in[0,1]$, the $\operatorname{DMU}\left(X^{*}, Y^{*}\right)=\gamma(\tilde{X}, \tilde{Y})+(1-\gamma)(\hat{X}, \hat{Y})$ satisfies (12) with the vectors $\lambda^{*}, \alpha^{*}$ and $\beta^{*}$ as defined below. First, let

$$
\lambda^{*}=\gamma \tilde{\lambda}+(1-\gamma) \hat{\lambda}
$$

Define $J^{+}=\left\{j \in J \mid \lambda_{j}^{*}>0\right\}$. For each $j \in J^{+}$, define $\beta_{j}^{*}$ and $\alpha_{j}^{*}$ from the equalities

$$
\begin{gathered}
\lambda_{j}^{*} \beta_{j}^{*}=\gamma \tilde{\lambda}_{j} \tilde{\beta}_{j}+(1-\gamma) \hat{\lambda}_{j} \hat{\beta}_{j}, \\
\lambda_{j}^{*} \beta_{j}^{*} \alpha_{j}^{*}=\gamma \tilde{\lambda}_{j} \tilde{\beta}_{j} \tilde{\alpha}_{j}+(1-\gamma) \hat{\lambda}_{j} \hat{\beta}_{j} \hat{\alpha}_{j} .
\end{gathered}
$$

For each $j \in J^{+}$, from (A.1) and (A.2), we have

$$
\beta_{j}^{*}=\frac{\gamma \tilde{\lambda}_{j} \tilde{\beta}_{j}+(1-\gamma) \hat{\lambda}_{j} \hat{\beta}_{j}}{\lambda_{j}^{*}}=\frac{\gamma \tilde{\lambda}_{j} \tilde{\beta}_{j}+(1-\gamma) \hat{\lambda}_{j} \hat{\beta}_{j}}{\gamma \tilde{\lambda}_{j}+(1-\gamma) \hat{\lambda}_{j}}
$$

Taking into account the last term of (A.4),

$$
\min \left\{\tilde{\beta}_{j}, \hat{\beta}_{j}\right\} \leq \beta_{j}^{*} \leq \max \left\{\tilde{\beta}_{j}, \hat{\beta}_{j}\right\}
$$

By (A.5), $\beta_{j}^{*} \geq 1, \forall j \in J^{+}$. Then similarly, from (A.2) and (A.3), we have

$$
\begin{gathered}
\alpha_{j}^{*}=\frac{\gamma \tilde{\lambda}_{j} \tilde{\beta}_{j} \tilde{\alpha}_{j}+(1-\gamma) \hat{\lambda}_{j} \hat{\beta}_{j} \hat{\alpha}_{j}}{\lambda_{j}^{*} \beta_{j}^{*}}=\frac{\gamma \tilde{\lambda}_{j} \tilde{\beta}_{j} \tilde{\alpha}_{j}+(1-\gamma) \hat{\lambda}_{j} \hat{\beta}_{j} \hat{\alpha}_{j}}{\gamma \tilde{\lambda}_{j} \tilde{\beta}_{j}+(1-\gamma) \hat{\lambda}_{j} \hat{\beta}_{j}}, \\
\min \left\{\tilde{\alpha}_{j}, \hat{\alpha}_{j}\right\} \leq \alpha_{j}^{*} \leq \max \left\{\tilde{\alpha}_{j}, \hat{\alpha}_{j}\right\} .
\end{gathered}
$$

From (A.1) and (A.3),

$$
\begin{gathered}
\beta_{j}^{*} \alpha_{j}^{*}=\frac{\gamma \tilde{\lambda}_{j} \tilde{\beta}_{j} \tilde{\alpha}_{j}+(1-\gamma) \hat{\lambda}_{j} \hat{\beta}_{j} \hat{\alpha}_{j}}{\lambda_{j}^{*}}=\frac{\gamma \tilde{\lambda}_{j} \tilde{\beta}_{j} \tilde{\alpha}_{j}+(1-\gamma) \hat{\lambda}_{j} \hat{\beta}_{j} \hat{\alpha}_{j}}{\gamma \tilde{\lambda}_{j}+(1-\gamma) \hat{\lambda}_{j}}, \\
\min \left\{\tilde{\beta}_{j} \tilde{\alpha}_{j}, \hat{\beta}_{j} \hat{\alpha}_{j}\right\} \leq \beta_{j}^{*} \alpha_{j}^{*} \leq \max \left\{\tilde{\beta}_{j} \tilde{\alpha}_{j}, \hat{\beta}_{j} \hat{\alpha}_{j}\right\} .
\end{gathered}
$$

Finally, for each $j$ such that $\lambda_{j}^{*}=0$ (i.e. $j \in J \backslash J^{+}$), arbitrarily define $\beta_{j}^{*}=\alpha_{j}^{*}=1$.

Let us prove that DMU $\left(X^{*}, Y^{*}\right)$ and vectors $\lambda^{*}, \alpha^{*}$ and $\beta^{*}$ satisfy (12). Consider (12.1) and (12.2). State each of these inequalities twice, with $\operatorname{DMU}(\tilde{X}, \tilde{Y})$ and vectors $\tilde{\lambda}, \tilde{\alpha}, \tilde{\beta}$, and with $\operatorname{DMU}(\hat{X}, \hat{Y})$ and vectors $\hat{\lambda}, \hat{\alpha}, \hat{\beta}$, respectively. Multiply the first inequality in each pair by $\gamma$ and the second by $1-\gamma$, and add the two resulting inequalities. Noting (A.3) and 
rearranging the terms, we obtain (12.1) and (12.2) stated for the DMU $\left(X^{*}, Y^{*}\right)$ with $\lambda^{*}$, $\alpha^{*}$ and $\beta^{*}$.

To prove (12.3)-(12.9), let $\lambda_{j}^{*}>0$. The case $\tilde{\lambda}_{j}=0$ or $\hat{\lambda}_{j}=0$ is trivial. For example, if $\tilde{\lambda}_{j}=0$, by (A.4) and (A.6), $\beta_{j}^{*}=\hat{\beta}_{j}, \alpha_{j}^{*}=\hat{\alpha}_{j}$, and inequalities (12.3)-(12.9) are true because $(\hat{X}, \hat{Y})$ satisfies $(12)$ with $\hat{\lambda}, \hat{\alpha}, \hat{\beta}$. Let both $\tilde{\lambda}_{j} \neq 0$ and $\hat{\lambda}_{j} \neq 0$. Then conditions $(12.3)$ and (12.4) are obviously satisfied.

Noting (3), conditions (12.5)-(12.9) are true for subvectors $Y^{D}, X^{D}, X^{U}, Y^{P}$ and $X^{P}$ together with each of the two sets of vectors: $\tilde{\lambda}, \tilde{\alpha}, \tilde{\beta}$, and $\hat{\lambda}, \hat{\alpha}, \hat{\beta}$. Consider (12.5). By (A.7), there exists a $\delta \in[0,1]$ such that $\alpha_{j}^{*}=\delta \tilde{\alpha}_{j}+(1-\delta) \hat{\alpha}_{j}$. State (12.5) twice, with $\tilde{\alpha}_{j}$ and $\hat{\alpha}_{j}$. Multiply the former by $\delta$ and the latter by $1-\delta$. By adding the resulting inequalities and rearranging the terms, observe that (12.5) is satisfied by $\alpha_{j}^{*}$. The inequality (12.6) is proved in the same way. The proof of inequalities (12.7)-(12.9) follows from (A.5) and (A.8).

Conditions (12.10)-(12.13) follow from (A.1), (A.5) and (A.7). Finally note that the $\operatorname{DMU}\left(X^{*}, Y^{*}\right)$ is nonnegative and satisfies $(2)$. Therefore, $\left(X^{*}, Y^{*}\right) \in \tilde{T}_{\mathrm{CRS}}^{\mathrm{R}}$.

Lemma 2. Technology $\tilde{T}_{\mathrm{CRS}}^{\mathrm{R}}$ satisfies Axioms 4 and 5 .

Proof of Lemma 2. Let $(X, Y)$ satisfy (2) and (12) with $\lambda, \alpha$ and $\beta$. Let $0 \leq \gamma<1$. Then the DMU $\left(X^{\gamma}, Y^{\gamma}\right)$ defined in Axiom 4 (here we use $\gamma$ instead of $\alpha$ ) satisfies (12) with the same $\lambda, \beta$ and $\tilde{\alpha}=\gamma \alpha$. Indeed, conditions (12.1)-(12.4), (12.7)-(12.13) are straightforward. Also, in (12.5),

$$
\begin{aligned}
\tilde{\alpha}_{j} Y_{j}^{D}+\left(1-\tilde{\alpha}_{j}\right) \bar{q} & =\gamma \alpha_{j} Y_{j}^{D}+\left(1-\gamma \alpha_{j}\right) \bar{q} \\
& =\gamma\left(\alpha_{j} Y_{j}^{D}+\left(1-\alpha_{j}\right) \bar{q}\right)-\gamma \bar{q}+\bar{q} \geq \gamma Y^{D}+(1-\gamma) \bar{q}=\left(Y^{\gamma}\right)^{D},
\end{aligned}
$$

and (12.5) follows. Inequality (12.6) is proved in a similar way. Conditions (2) are straightforward.

The proof of Axiom 5 is similar. Let $\gamma>1$. Then the $\operatorname{DMU}\left(X^{\gamma}, Y^{\gamma}\right)$ defined in Axiom 5 (we use $\gamma$ instead of $\alpha$ ) satisfies conditions (2) and (12) with the same $\lambda, \alpha$ and $\tilde{\beta}=\gamma \beta$.

Lemma 3. Technology $\tilde{T}_{\mathrm{CRS}}^{\mathrm{R}}$ is a subset of any technology $T^{\prime}$ that satisfies Axioms 1-5.

Proof of Lemma 3. Assume that $(X, Y) \in \tilde{T}_{\mathrm{CRS}}^{\mathrm{R}}$. Therefore, $(X, Y)$ satisfies (2) and (12) with some $\lambda, \alpha$ and $\beta$. We need to prove that $(X, Y) \in T^{\prime}$, where $T^{\prime}$ is any technology that satisfies Axioms 1-5. By Axiom 1, all observed DMUs $\left(X_{j}, Y_{j}\right) \in T^{\prime}, \forall j \in J$.

Without loss of generality, let $\lambda_{j}>0$ for all $j \in J$ and, therefore, (12.3)-(12.9) are unconditionally true. Consider the DMUs $\left(\tilde{X}_{j}, \tilde{Y}_{j}\right)$ constructed from $\left(X_{j}, Y_{j}\right) \in T^{\prime}$ using the components on the left-hand side of inequalities (12) and the vector $\overline{\min }\left\{\beta_{j}\left(\alpha_{j} Y_{j}^{P}\right), \bar{Y}^{P}\right\}$ defined in $\S 5.1$ :

$$
\left(\begin{array}{c}
\tilde{X}_{j} \\
\tilde{Y}_{j}
\end{array}\right)=\left(\begin{array}{c}
\beta_{j}\left(\alpha_{j} X_{j}^{V}\right), X_{j}^{F}, \alpha_{j} X_{j}^{D}+\left(1-\alpha_{j}\right) \bar{p}, \beta_{j} X^{U}, \beta_{j}\left(\alpha_{j} X_{j}^{P}\right) \\
\beta_{j}\left(\alpha_{j} Y_{j}^{V}\right), Y_{j}^{F}, \alpha_{j} Y_{j}^{D}+\left(1-\alpha_{j}\right) \bar{q}, \overline{\min }\left\{\beta_{j}\left(\alpha_{j} Y_{j}^{P}\right), \bar{Y}^{P}\right\}
\end{array}\right) .
$$

The DMU $\left(\tilde{X}_{j}, \tilde{Y}_{j}\right)$ is the result of contraction of the observed DMU $\left(X_{j}, Y_{j}\right) \in T^{\prime}$ with $0 \leq \alpha_{j} \leq 1$, and its further expansion with $\beta_{j} \geq \underset{\sim}{1}$, following the "rules" of Axioms 4-5. By $(12.3)-(12.9)$ and because $(X, Y)$ satisfies $(2),\left(\tilde{X}_{j}, \tilde{Y}_{j}\right)$ also satisfies (2). By Axioms 4 and $5,\left(\tilde{X}_{j}, \tilde{Y}_{j}\right) \in T^{\prime}$.

Change the ratio inputs and outputs of the DMU $\left(\tilde{X}_{j}, \tilde{Y}_{j}\right)$ to the corresponding equal or "worse" values $X^{F}, X^{D}, X^{U}, X^{P}$ and $Y^{F}, Y^{D}, Y^{P}$ on the right-hand side of (12.3)-(12.9). Note that, by (2) and (12.8), $\overline{\min }\left\{\beta_{j}\left(\alpha_{j} Y_{j}^{P}\right), \bar{Y}^{P}\right\} \geq Y^{P}$. Define

$$
\left(\hat{X}_{j}, \hat{Y}_{j}\right)=\left(\beta_{j}\left(\alpha_{j} X_{j}^{V}\right), X^{F}, X^{D}, X^{U}, X^{P}, \beta_{j}\left(\alpha_{j} Y_{j}^{V}\right), Y^{F}, Y^{D}, Y^{P}\right) .
$$


By Axiom 2, $\left(\hat{X}_{j}, \hat{Y}_{j}\right) \in T^{\prime}$. All DMUs $\left(\hat{X}_{j}, \hat{Y}_{j}\right), j \in J$, have the same subvectors of ratio inputs and outputs and, by Axiom 3, the following DMU is in $T^{\prime}$ :

$$
(\hat{X}, \hat{Y})=\left(\sum_{j \in J} \lambda_{j} \beta_{j}\left(\alpha_{j} X_{j}^{V}\right), X^{F}, X^{D}, X^{U}, X^{P}, \sum_{j \in J} \lambda_{j} \beta_{j}\left(\alpha_{j} Y_{j}^{V}\right), Y^{F}, Y^{D}, Y^{P}\right) .
$$

Finally, by (12.1) and (12.2), $(\hat{X}, \hat{Y})$ dominates $(X, Y)$. By Axiom $2,(X, Y) \in T^{\prime}$.

Proof of Theorem 3. The proof follows from Lemmas 4 and 5.

Lemma 4. Let $D M U(X, Y)$ satisfy conditions (12) with $\lambda, \alpha, \beta \in \mathbb{R}^{n}$. Then $(X, Y)$ satisfies conditions (13) with the same vector $\lambda$ and some vectors $\mu, \nu \in \mathbb{R}^{n}$.

Proof of Lemma 4. For all $j \in J$, define

$$
\begin{gathered}
\mu_{j}=\lambda_{j} \alpha_{j}\left(\beta_{j}-1\right), \\
\nu_{j}=\lambda_{j}\left(1-\alpha_{j}\right) .
\end{gathered}
$$

From (A.9) and (A.10), and taking into account (12.11) and (12.12), for all $j \in J$, we have

$$
\begin{gathered}
\lambda_{j}+\mu_{j}-\nu_{j}=\lambda_{j} \beta_{j} \alpha_{j}, \\
\lambda_{j}-\nu_{j}=\lambda_{j} \alpha_{j}, \\
\text { if } \mu_{j}>0 \text { then } \lambda_{j}>0 .
\end{gathered}
$$

Conditions (13.1) and (13.2) follow by substituting (A.11) into (12.1) and (12.2). By (A.13), $\lambda_{j}+\mu_{j}>0$ implies $\lambda_{j}>0$, and (13.3) and (13.4) follow from (12.3) and (12.4). To prove (13.5a), (13.6a), (13.8a) and (13.9), multiply both sides of (12.5), (12.6), (12.8) and (12.9) by $\lambda_{j}$. To prove (13.7a), multiply (12.7) by $\lambda_{j} \alpha_{j}$. The proof of all these conditions is completed by noting (A.10)-(A.13). Furthermore, (12.5) and (12.6) imply (13.5b) and (13.6b). Indeed, by (A.13) and Data Assumption 3,

$$
\begin{aligned}
& Y_{j}^{D} \geq \alpha_{j} Y_{j}^{D}+\left(1-\alpha_{j}\right) \bar{q} \geq Y^{D} \\
& X_{j}^{D} \leq \alpha_{j} X_{j}^{D}+\left(1-\alpha_{j}\right) \bar{p} \leq X^{D} .
\end{aligned}
$$

Similarly, (12.7) implies (13.7b). Indeed, $X_{j}^{U} \leq \beta_{j} X_{j}^{U} \leq X^{U}$.

Let us prove (13.8b). Consider any $j \in J$ and $r \in \mathrm{O}^{P}$ such that $\mu_{j}>0$ and $Y_{r}^{P}>0$. Then by (A.13), $\lambda_{j}>0$. Then (12.8) is true and, in particular, for the given $r$, we have

$$
\beta_{j} \alpha_{j} Y_{j r}^{P} \geq Y_{r}^{P}
$$

Because, as assumed, $Y_{r}^{P}>0$, we have $Y_{j r}^{P}>0$, and (13.8b) follows. Finally, (12.10)-(12.13) imply (13.10)-(13.12).

Lemma 5. Let $\operatorname{DMU}(X, Y)$ satisfy conditions (13) with vectors $\lambda, \mu, \nu \in \mathbb{R}^{n}$. Then $(X, Y)$ satisfies conditions (12) with some vectors $\tilde{\lambda}, \tilde{\alpha}, \tilde{\beta} \in \mathbb{R}^{n}$.

Proof of Lemma 5. Below we identify five different types of $j \in J$ depending on the values of $\lambda_{j}, \mu_{j}$ and $\nu_{j}$. Each type requires a different definition of vectors $\tilde{\lambda}_{j}, \alpha_{j}$ and $\beta_{j}$ that satisfy (12.3)-(12.9) and (12.11)-(12.13). Regardless of the type, for each $j$, we have

$$
\lambda_{j}+\mu_{j}-\nu_{j}=\tilde{\lambda}_{j} \alpha_{j} \beta_{j} .
$$


This implies that conditions (12.1) and (12.2) follow from (13.1) and (13.2). Finally, the normalizing equality (12.10) may not be true for the vector $\tilde{\lambda}$ but becomes true after a renormalization of $\tilde{\lambda}$.

Consider the following five mutually exclusive types of $j \in J$.

Type 1. This type includes all $j \in J: \lambda_{j}=\mu_{j}=\nu_{j}=0$. Define $\tilde{\lambda}_{j}=0$ and arbitrarily let $\alpha_{j}=\beta_{j}=1$. The only conditions that need proving are (12.11)-(12.13). These are obviously true.

Type 2. This type includes all $j \in J: \lambda_{j}-\nu_{j}>0$. By (13.11) this implies $\lambda_{j}>0$. Define

$$
\begin{aligned}
\tilde{\lambda}_{j} & =\lambda_{j}, \\
\alpha_{j} & =\left(\lambda_{j}-\nu_{j}\right) / \lambda_{j}, \\
\beta_{j} & =\left(\lambda_{j}+\mu_{j}-\nu_{j}\right) /\left(\lambda_{j}-\nu_{j}\right) .
\end{aligned}
$$

From (A.16) and (A.17),

$$
\beta_{j} \alpha_{j}=\left(\lambda_{j}+\mu_{j}-\nu_{j}\right) / \lambda_{j}
$$

Note that $\alpha_{j}$ and $\beta_{j}$ satisfy (12.11)-(12.13). Because $\tilde{\lambda}_{j}=\lambda_{j}>0$, we need to prove (12.3)-(12.9). Note that (13.3) and (13.4) imply (12.3) and (12.4). Inequalities (12.5) and (12.6) follow by dividing both sides of (13.5a) and (13.6a) by $\lambda_{j}>0$ and noting (A.16). Condition (12.7) is obtained by dividing both sides of (13.7a) by $\lambda_{j}-\nu_{j}>0$ and noting (A.17). Inequalities (12.8) and (12.9) are obtained by dividing both sides of (13.8a) and (13.9) by $\lambda_{j}>0$ and noting (A.18).

Type 3. This type includes all $j \in J: \lambda_{j}=\nu_{j}>0$ (therefore, $\lambda_{j}-\nu_{j}=0$ ) and $\mu_{j}=0$. Define

$$
\begin{aligned}
\tilde{\lambda}_{j} & =\lambda_{j}>0, \\
\alpha_{j} & =0, \\
\beta_{j} & =1 .
\end{aligned}
$$

Note that $\alpha_{j}$ and $\beta_{j}$ satisfy (12.11)-(12.13). Because $\tilde{\lambda}_{j}=\lambda_{j}>0$, we need to prove (12.3)(12.9). Note that (12.3) and (12.4) follow from (13.3) and (13.4). To prove (12.5) and (12.6), divide both sides of (13.5a) and (13.6a) by $\lambda_{j}$ and note that $\left(\lambda_{j}-\nu_{j}\right) / \lambda_{j}=0=\alpha_{j}$ and $\nu_{j} / \lambda_{j}=1=1-\alpha_{j}$. Because $\lambda_{j}>0$ and noting (A.21), condition (13.7b) implies (12.7).

For any $j$ of Type $3, \lambda_{j}+\mu_{j}-\nu_{j}=0$. Because $\lambda_{j}>0$, (13.8a) implies $Y^{P}=0$, and (12.8) is true. From (A.20) and (A.21), $\beta_{j} \alpha_{j}=0$, and (12.9) is trivially true. Define

Type 4. This type includes all $j \in J: \lambda_{j}=\nu_{j}>0$ (therefore, $\lambda_{j}-\nu_{j}=0$ ) and $\mu_{j}>0$.

$$
\begin{aligned}
\tilde{\lambda}_{j} & =\lambda_{j}>0, \\
\alpha_{j} & =\varepsilon, \\
\beta_{j} & =\mu_{j} /\left(\lambda_{j} \varepsilon\right)=\mu_{j} /\left(\lambda_{j} \alpha_{j}\right),
\end{aligned}
$$

where $\varepsilon \in(0,1)$ is so small that, in (A.24) $\beta_{j} \geq 1$. Then conditions (12.11)-(12.13) are true.

Because $\tilde{\lambda}_{j}=\lambda_{j}>0,(13.3)$ and (13.4) imply (12.3) and (12.4). Divide both sides of (13.5a) and (13.6a) by $\lambda_{j}>0$. Noting that $\lambda_{j}-\nu_{j}=0$ and $\nu_{j} / \lambda_{j}=1$, and by Data Assumption 3, we have

$$
\begin{gathered}
\varepsilon Y_{j}^{D}+(1-\varepsilon) \bar{q} \geq 0 Y_{j}^{D}+1 \bar{q} \geq Y^{D}, \\
\varepsilon X_{j}^{D}+(1-\varepsilon) \bar{p} \leq 0 X_{j}^{D}+1 \bar{p} \leq X^{D} .
\end{gathered}
$$


Taking into account (A.23), the inequalities (A.25) and (A.26) become (12.5) and (12.6).

If $\mathrm{I}^{U} \neq \varnothing$ then, as $\lambda_{j}-\nu_{j}=0$ and $\mu_{j}>0$, from (13.7a), $X_{j}^{U}=0$, and (12.7) follows. Finally, (12.8) and (12.9) follow by dividing (13.8a) and (13.9) by $\lambda_{j}>0$, noting that $\lambda_{j}-\nu_{j}=0$ and by (A.24).

Type 5. This type includes all $j \in J: \lambda_{j}=\nu_{j}=0$ and $\mu_{j}>0$. For all such $j,(13.8 \mathrm{~b})$ implies there exists a $\beta_{j} \geq 1$ such that $\beta_{j} Y_{j}^{P} \geq Y^{P}$, and (12.8) follows. Also define

$$
\begin{aligned}
& \alpha_{j}=1, \\
& \tilde{\lambda}_{j}=\mu_{j} /\left(\alpha_{j} \beta_{j}\right)=\mu_{j} / \beta_{j}>0 .
\end{aligned}
$$

Note that $\alpha_{j}$ and $\beta_{j}$ satisfy (12.11)-(12.13). Also note that $\tilde{\lambda}_{j}$ can be larger than 1 . Below we renormalize vector $\tilde{\lambda}$ to correct this. Because $\tilde{\lambda}_{j}>0$, we need to prove (12.3)(12.7) and (12.9). By (A.28), $\mu_{j}>0$. Then (13.3) and (13.4) imply (12.3) and (12.4), and conditions (13.5b) and (13.6b) imply (12.5) and (12.6), where $\alpha_{j}=1$ as in (A.27). Because $\mu_{j}>0$ and by (13.7a) and (13.9), if $\mathrm{I}^{U} \neq \varnothing$ then $X_{j}^{U}=\mathbf{0}$, and if $\mathrm{I}^{P} \neq \varnothing$ then $X_{j}^{P}=\mathbf{0}$. This implies (12.7) and (12.9).

We have proved that $(X, Y)$ satisfies (12.3)-(12.9) with the vectors $\tilde{\lambda}, \alpha$ and $\beta$ whose components are defined differently depending on the type of $j$. It is easy to verify that equality (A.14) is true for each type of $j$. Therefore, (13.1) and (13.2) imply (12.1) and $(12.2)$.

Finally, consider the normalizing equality (12.10). If all $j \in J$ are of Types $1-4$ only, then from (A.15), (A.19), (A.22), we have $\tilde{\lambda}=\lambda$, and (12.10) follows. Assume there exists a $j^{*}$ of Type 5. Because $\lambda_{j^{*}}=0$ and, by (A.28), $\tilde{\lambda}_{j^{*}}>0$, we have $\mathbf{1}^{\top} \tilde{\lambda}=\Lambda^{*}>1$. Let $\hat{\lambda}=\tilde{\lambda} / \Lambda^{*}, \hat{\beta}=\beta \Lambda^{*}$ and $\hat{\alpha}=\alpha$, where $\tilde{\lambda}, \beta$ and $\alpha$ are the vectors whose components were defined above for each type of $j$. Then all conditions (12) are true with $\hat{\lambda}, \hat{\alpha}$ and $\hat{\beta}$. (As shown above, in this case we have $X_{j}^{U}=\mathbf{0}$ and $X_{j}^{P}=\mathbf{0}$.)

Proof of Proposition 2. Consider the statement of $T_{\mathrm{CRS}}^{\mathrm{R}}$ by Theorem 3. Assume that a sequence of DMUs $\left(X^{k}, Y^{k}\right) \in T_{\mathrm{CRS}}^{\mathrm{R}}$ converges to $\left(X^{*}, Y^{*}\right) \in \mathbb{R}^{m} \times \mathbb{R}^{s}$ as $k \rightarrow+\infty$. We need to prove that $\left(X^{*}, Y^{*}\right) \in T_{\mathrm{CRS}}^{\mathrm{R}}$. For sufficiently large $k$, all vectors $X^{k}$ are in some small neighborhood of vector $X^{*}$. For each $k,\left(X^{k}, Y^{k}\right)$ satisfies (13) with some vectors $\lambda^{k}, \mu^{k}$ and $\nu^{k}$. Because of (13.10)-(13.12), all vectors $\lambda^{k}, \nu^{k} \in[0,1]^{n}$, and $\lambda_{j}^{k}>0$ for at least one $j$. Data Assumption 4, (13.2), (13.7a) and (13.9) imply that $\mu_{j}^{k}$ cannot be unbounded, and therefore there exists a constant $M_{1}>0$ such that $\mu_{j}^{k} \leq M$, for all $j \in J$ and $k=1,2, \ldots$ Therefore, all vectors $\mu^{k} \in[0, M]^{n}$.

The sequence $\left\{\left(\lambda^{k}, \mu^{k}, \nu^{k}\right)\right\}$ is in the set $\Delta=[0,1]^{n} \times[0,1]^{n} \times[0, M]^{n}$. Because $\Delta$ is a compact set, there exists a subsequence of this sequence that converges to some $\left(\lambda^{*}, \mu^{*}, \nu^{*}\right) \in$ $\Delta$. Without loss of generality, let $\left\{\left(\lambda^{k}, \mu^{k}, \nu^{k}\right)\right\} \rightarrow\left(\lambda^{*}, \mu^{*}, \nu^{*}\right)$ when $k \rightarrow+\infty$.

Consider conditions (13), with the exception of (13.8b) which is dealt with below. By stating these conditions with the DMU $\left(X^{k}, Y^{k}\right)$ and components $\lambda_{j}^{k}, \mu_{j}^{k}, \nu_{j}^{k}$, and taking $k$ to $+\infty$, we prove that these are satisfied at the limit by $\left(X^{*}, Y^{*}\right)$ and $\lambda^{*}, \mu^{*}$ and $\nu^{*}$. Assume that, for some $r \in \mathrm{O}^{P}, \mu_{j}^{*}>0$ and $\left(Y^{*}\right)_{r}^{P}>0$. Then, for all sufficiently large $k, \mu_{j}^{k}>0$ and $\left(Y^{k}\right)_{r}^{P}>0$. By (13.8b), $Y_{j r}^{P}>0$. Therefore, $(13.8 \mathrm{~b})$ is true for $\left(X^{*}, Y^{*}\right)$ and $\mu^{*}$.

Finally, all DMUs $\left(X^{k}, Y^{k}\right)$, and therefore the limit DMU $\left(X^{*}, Y^{*}\right)$, satisfy $(2)$.

Proof of Proposition 3. Any DMU $(X, Y)$ that satisfies (4) with some $\lambda$, also satisfies (12) with the same $\lambda$ and $\alpha=\beta=\mathbf{1}$, and the proof follows.

Proof of Proposition 4. If DMU (X,Y) satisfies (12.1)-(12.4) and (12.10)-(12.13) with some $\lambda_{j}, \alpha_{j}$ and $\beta_{j}, \forall j \in J$, then it satisfies (4.1)-(4.4) and (4.6) with $\tilde{\lambda}_{j}=\lambda_{j} \alpha_{j} \beta_{j}$. In 
particular, if $\tilde{\lambda}_{j}>0$ then $\lambda_{j}>0$. Then (4.3) and (4.4) follow from (12.3) and (12.4). Conversely, if $(X, Y)$ satisfies (4.1)-(4.4) and (4.6) with some $\lambda$, define $\Lambda=\sum_{j \in J} \lambda_{j}$. Because $Y_{\tilde{\beta}}^{V} \neq \mathbf{0}$ and by (4.1), $\Lambda>0$. For all $j \in J$, let $\tilde{\lambda}_{j}=\lambda_{j} / \Lambda$. Also, if $\Lambda \leq 1$, define $\tilde{\alpha}_{j}=\Lambda$ and $\tilde{\beta}_{j}=1, \forall j \in J$. Otherwise, i.e. if $\Lambda>1$, define $\tilde{\alpha}_{j}=1$ and $\tilde{\beta}_{j}=\Lambda, \forall j \in J$. Then conditions (12.1), (12.2), and (12.10)-(12.13) are true. Finally, because $\tilde{\alpha}_{j}>0$ and $\tilde{\beta}_{j}>0$ for all $j$, $\lambda_{j}>0$ implies $\tilde{\lambda}_{j}>0$ and, therefore, (4.3) and (4.4) imply (12.3) and (12.4). Therefore, $(X, Y)$ satisfies $(12.1)-(12.4)$ and (12.10)-(12.13) with $\tilde{\lambda}, \tilde{\alpha}$ and $\tilde{\beta}$.

In the proof of Propositions 5-7 we use R-CRS models $\tilde{\mathbb{M}}_{\text {CRS }}^{1}$ and $\tilde{\mathbb{M}}_{\text {CRS }}^{2}$. These are, respectively, equivalent to models $\mathbb{M}_{\text {CRS }}^{1}$ and $\mathbb{M}_{\text {CRS }}^{2}$ and are obtained from the latter by replacing their conditions (13) by the equivalent nonlinear conditions (12). We also require the following assumption that replaces the stronger Data Assumption 5 stated in $§ 6.2$.

Data Assumption 5*. (a) For model $\mathbb{M}_{\text {VRS }}^{1}$, there exists an $i^{*} \in \mathrm{I}^{V} \cap \mathrm{I}^{\theta}$ such that $X_{\text {oi }^{*}}^{V}>0$ and $X_{j i^{*}}^{V}>0, \forall j \in J$. (b) For model $\mathbb{M}_{\mathrm{VRS}}^{2}$, there exists an $r^{*} \in \mathrm{O}^{V} \cap \mathrm{O}^{\eta}$ such that $Y_{o r^{*}}^{V}>0$. (c) For model $\mathbb{M}_{\text {CRS }}^{1}$ (and $\tilde{\mathbb{M}}_{\text {CRS }}^{1}$ ), condition (a) is true and there exists an $r^{*} \in \mathrm{O}^{V}$ such that $Y_{o r^{*}}^{V}>0$. (d) For model $\mathbb{M}_{\mathrm{CRS}}^{2}$ (and $\tilde{\mathbb{M}}_{\mathrm{CRS}}^{2}$ ), condition (b) is true and there exists an $i^{*} \in \mathrm{I}^{V}$ such that $X_{o i^{*}}^{V}>0$ and $X_{j i^{*}}^{V}>0, \forall j \in J$.

Proof of Proposition 5. Consider model $\mathbb{M}_{\mathrm{VRS}}^{1}$. By Data Assumption $5^{*}(\mathrm{a})$ and (4.2), at any feasible solution to $\mathbb{M}_{\mathrm{VRS}}^{1}, \theta \geq 0$. Therefore, the infimum $\theta_{\mathrm{VRS}}^{*} \geq 0$. Because $T_{\mathrm{VRS}}^{\mathrm{R}}$ is a closed set, $\left(X_{o}\left(\theta_{\mathrm{VRS}}^{*}\right), Y_{o}\right) \in T_{\mathrm{VRS}}^{\mathrm{R}}$ and $\theta_{\mathrm{VRS}}^{*}$ is attained for some vector $\lambda^{*}$. By Data Assumption $5^{*}(\mathrm{a}), \sum_{j \in J} \lambda_{j}^{*} X_{j i^{*}}^{V}>0$. Because $X_{o i^{*}}^{V}>0$, we have $\theta_{\mathrm{VRS}}^{*}>0$. Consider model $\mathbb{M}_{\text {VRS }}^{2}$. Because of (4.5) and (4.6), the left-hand side of (4.1) is bounded above. By Data Assumption $5^{*}(\mathrm{~b})$, the supremum $\eta_{\mathrm{VRS}}^{*}$ is finite. Because $T_{\mathrm{VRS}}^{\mathrm{R}}$ is a closed set, $\eta_{\mathrm{VRS}}^{*}$ is attained and, obviously, $\eta_{\text {VRS }}^{*} \geq 1$.

Consider model $\tilde{\mathbb{M}}_{\mathrm{CRS}}^{1}$. Similar to the case of $\mathbb{M}_{\mathrm{VRS}}^{1}$, the infimum $\theta_{\mathrm{CRS}}^{*} \geq 0$ and is attained. Assume that $\theta_{\mathrm{CRS}}^{*}=0$. Then (12.2) and Data Assumption $5^{*}(\mathrm{a})$ imply $\lambda_{j} \alpha_{j} \beta_{j}=0, \forall j \in J$. Then by $(12.1), Y_{o}^{V}=\mathbf{0}$, which contradicts Data Assumption $5^{*}(\mathrm{c})$. Therefore, $\theta_{\mathrm{CRS}}^{*}>0$.

Consider model $\tilde{\mathbb{M}}_{\text {CRS }}^{2}$. By (12.2) and Data Assumption $5^{*}(\mathrm{~d})$, there exists a $\delta$ such that $\lambda_{j} \alpha_{j} \beta_{j} \leq \delta, \forall j \in J$. Then the left-hand side of (12.1) is bounded above. By Data Assumption $5^{*}(\mathrm{~b})$, the supremum $\eta_{\mathrm{CRS}}^{*}$ is finite and, obviously, $\eta_{\mathrm{CRS}}^{*} \geq 1$. Because $T_{\mathrm{CRS}}^{\mathrm{R}}$ is a closed set, $\eta_{\mathrm{CRS}}^{*}$ is attained.

By Proposition 3, $T_{\mathrm{VRS}}^{\mathrm{R}} \subset T_{\mathrm{CRS}}^{\mathrm{R}}$. Therefore, $\theta_{\mathrm{CRS}}^{*} \leq \theta_{\mathrm{VRS}}^{*}$ and $\eta_{\mathrm{CRS}}^{*} \geq \eta_{\mathrm{VRS}}^{*}$.

Proof of Proposition 6. Let $\lambda^{*}, \alpha^{*}, \beta^{*}$, and $\theta^{*}$ be optimal in model $\tilde{\mathbb{M}}_{\mathrm{CRS}}^{1}$. By Proposition 5, $\theta^{*}>0$. Then $\lambda^{*}, \alpha^{*}, \beta=\beta^{*} / \theta^{*}$, and $\eta=1 / \theta^{*}$ is feasible in model $\tilde{\mathbb{M}}_{\text {CRS }}^{2}$. Because $E_{\mathrm{CRS}}^{1}\left(X_{o}, Y_{o}\right)=\theta^{*}$ and $E_{\mathrm{CRS}}^{2}\left(X_{o}, Y_{o}\right) \leq 1 / \eta=\theta^{*}$, it follows that $E_{\mathrm{CRS}}^{1}\left(X_{o}, Y_{o}\right) \geq$ $E_{\text {CRS }}^{2}\left(X_{o}, Y_{o}\right)$. Conversely, let $\lambda^{*}, \alpha^{*}, \beta^{*}$, and $\eta^{*}$ be optimal in model $\tilde{\mathbb{M}}_{\text {CRS }}^{2}$. Then $\eta^{*} \geq 1$, and $\lambda^{*}, \alpha=\alpha^{*} / \eta^{*}, \beta^{*}$, and $\theta=1 / \eta^{*}$ is feasible in model $\tilde{\mathbb{M}}_{\mathrm{CRS}}^{1}$. Therefore, $E_{\mathrm{CRS}}^{1}\left(X_{o}, Y_{o}\right) \leq$ $E_{\mathrm{CRS}}^{2}\left(X_{o}, Y_{o}\right)$, and $E_{\mathrm{CRS}}^{1}\left(X_{o}, Y_{o}\right)=E_{\mathrm{CRS}}^{2}\left(X_{o}, Y_{o}\right)$.

Proof of Proposition 7. We need to consider only the inputs and outputs that change in the process of minimization or maximization. (a) By Proposition 5, in both models $\mathbb{M}_{\text {VRS }}^{1}$ and $\tilde{\mathbb{M}}_{\text {CRS }}^{1}, \theta^{*} \leq 1$ and, therefore, $X_{o}^{R}\left(\theta^{*}\right) \leq X_{o}^{R} \leq \bar{X}^{R}$. At any feasible solution to $\mathbb{M}_{\text {VRS }}^{2}$, there exists a $j^{*}$ such that $\lambda_{j^{*}}>0$. Because all observed DMUs $j \in J$ satisfy (2), condition (4.3) implies $Y_{o}^{R}(\eta) \leq Y_{j^{*}}^{R} \leq \bar{Y}^{R}$. Therefore, conditions (2) are satisfied at any optimal solution to the above three models.

(b) At any feasible solution to $\tilde{\mathbb{M}}_{\text {CRS }}^{2}$, there exists a $j^{*}$ such that $\lambda_{j^{*}}>0$. By Data Assumption 2, (12.3) implies $Y_{o}^{F}(\eta) \leq Y_{j^{*}}^{F} \leq \bar{Y}^{F}$, and (12.5) implies $Y_{o}^{D}(\eta) \leq \alpha_{j^{*}} Y_{j}^{D}+(1-$ $\left.\alpha_{j^{*}}\right) \bar{q} \leq \bar{Y}^{D}$. 
Proof of Proposition 8. Let $u, v, \delta_{j}$ and $\varepsilon_{j}, j \in J$, be the dual vectors of appropriate dimensions, and $w$ the dual scalar that correspond to constraints (4.1)-(4.5), respectively. Then the dual to $\mathbb{M}_{\text {VRS }}^{1}$ takes on the form:

$$
\max u^{\top} Y_{o}^{V}+w
$$

subject to

$$
\begin{aligned}
& v^{\top} X_{o}^{V}=1, \\
& u^{\top} Y_{j}^{V}-v^{\top} X_{j}^{V}+w+\delta_{j}^{\top}\left(Y_{j}^{R}-Y_{o}^{R}\right)+\varepsilon_{j}^{\top}\left(-X_{j}^{R}+X_{o}^{R}\right) \leq 0, \quad \forall j \in J, \\
& u, v, \delta_{j}, \varepsilon_{j} \geq \mathbf{0}, \forall j \in J, w \text { sign free. }
\end{aligned}
$$

If $j \in J \backslash J_{o}$, then there exists a $r^{*} \in \mathrm{O}^{R}$ such that $Y_{j r^{*}}^{R}-Y_{o r^{*}}^{R}<0$ or there exists an $i^{*} \in \mathrm{I}^{R}$ such that $-X_{j i^{*}}^{R}+X_{o i^{*}}^{R}<0$. Then each inequality in (A.29.3) for $j \in J \backslash J_{o}$ is satisfied by taking a sufficiently large component $\delta_{j r^{*}}>0$ or $\varepsilon_{j i^{*}}>0$. Therefore, all inequalities (A.29.3) for $j \in J \backslash J_{o}$ are redundant and can be omitted from (A.29).

Let us show that the remaining inequalities (A.29.3) for all $j \in J_{o}$ can equivalently be stated by omitting the terms $\delta_{j}^{\top}\left(Y_{j}^{R}-Y_{o}^{R}\right)$ and $\varepsilon_{j}^{\top}\left(-X_{j}^{R}+X_{o}^{R}\right)$, which leads to program (14). Indeed, any feasible solution $(u, v, w)$ in (14) is also feasible in (A.29) with $\delta_{j}=\mathbf{0}$ and $\varepsilon_{j}=\mathbf{0}$, $j \in J_{o}$. Conversely, let $(\hat{u}, \hat{v}, \hat{w})$ be feasible in (A.29) with some $\delta_{j} \geq \mathbf{0}$ and $\varepsilon_{j} \geq \mathbf{0}, j \in J_{o}$. For each $j \in J_{o}$ we have $Y_{j}^{R}-Y_{o}^{R} \geq \mathbf{0}$ and $-X_{j}^{R}+X_{o}^{R} \geq \mathbf{0}$. Therefore, $(\hat{u}, \hat{v}, \hat{w})$ is feasible in (A.29) with $\delta_{j}=\mathbf{0}$ and $\varepsilon_{j}=\mathbf{0}$, for all $j \in J_{o}$. Therefore, $(\hat{u}, \hat{v}, \hat{w})$ is feasible in (14). 


\section{References}

Banker, R.D., Charnes, A., Cooper, W.W. (1984) Some models for estimating technical and scale efficiencies in data envelopment analysis. Management Science, 30(9), 1078-1092.

Banker, R.D., Morey, R.C. (1986a) Efficiency analysis for exogenously fixed inputs and outputs. Operations Research, 34(4), 513-521.

Banker, R.D., Morey, R.C. (1986b) The use of categorical variables in data envelopment analysis. Management Science, 32(12), 1613-1627.

Chambers, R.G., Chung, Y., Färe, R. (1998) Profit, directional distance functions, and Nerlovian efficiency. Journal of Optimization Theory and Applications, 98(2), 351-364.

Charnes, A., Cooper, W.W., Rhodes, E. (1978) Measuring the efficiency of decision making units. European Journal of Operational Research, 2(6), 429-444.

Cooper, W.W., Seiford, L.M., Tone, K. (2007) Data envelopment analysis. A comprehensive text with models, applications, references and DEA-Solver software. 2nd Edition. New York: Springer Science + Busines Media.

Deprins, D., Simar, L., Tulkens, H. (1984) Measuring labor-efficiency in post offices. In M. Marchand, P. Pestieau, H. Tulkens (Eds.), The performance of public enterprises: concepts and measurements (pp. 243267). Amsterdam: North-Holland.

Dulá, J.H. (2009) A geometrical approach for generalizing the production possibility set in DEA. Journal of the Operational Research Society, 60(11), 1546-1555.

Dyson, R.G., Allen, R., Camanho, A.S., Podinovski, V.V., Sarrico, C., Shale, E.A. (2001) Pitfalls and protocols in DEA. European Journal of Operational Research, 132(2), 245-259.

Emrouznejad, A., Amin, G.R. (2009) DEA models for ratio data: convexity consideration. Applied Mathematical Modelling, 33(1), 486-498.

Gaynor, M., Seider, H., Vogt, W.B. (2005) The volume-outcome effect, scale economies, and learning-bydoing. American Economic Review, 95(2), 243-247.

Golany, B., Thore, S. (1997) The economic and social performance of nations: efficiency and returns to scale. Socio-Economic Planning Sciences, 31(3), 191-204.

Hollingsworth, B., Smith, P. (2003) Use of ratios in data envelopment analysis. Applied Economics Letters, 10(11), 733-735

Olesen, O.B., Petersen, N.C. (1995) Incorporating quality into data Envelopment analysis: a stochastic dominance Aapproach. International Journal of Production Economics, 39(1-2), 117-135.

Olesen, O.B., Petersen, N.C. (2006) Controlling for socioeconomic characteristics in DEA. North American Productivity Workshop, Stern School of Business, New York University, NY.

Olesen, O.B., Petersen, N.C. (2009) Target and technical efficiency in DEA - controlling for environmental characteristics. Journal of Productivity Analysis, 32(1), 27-40.

Paradi, J.C., Asmild, M., Simak, P.C. (2004a) Using DEA and worst practice DEA in credit risk evaluation. Journal of Productivity Analysis, 21(2), 153-165.

Paradi, J.C., Vela, S., Yang, Z. (2004b) Assessing bank and bank branch performance. In W.W. Cooper, L.M. Seiford, J. Zhu (Eds.), Handbook on data envelopment analysis (pp. 349-400). Boston: Kluwer Academic Publishers.

Paradi, J.C., Vela, S.A., Zhu, H. (2009) Adjusting for cultural differences, a new DEA model applied to a merged bank. Journal of Productivity Analysis, 33(2), 109-123.

Podinovski, V.V. (2005) Selective convexity in DEA models. European Journal of Operational Research, $161(2), 552-563$

Ruggiero, J. (1996) On the measurement of technical efficiency in the public sector. European Journal of Operational Research, 90(3), 553-565.

Simar, L., Wilson, P.W. (2002) Non-parametric tests of returns to scale. European Journal of Operational Research, 139(1), 115-132.

Thanassoulis, E., Boussofiane, A., Dyson, R.G. (1995) Exploring output quality targets in the provision of perinatal care in England using data envelopment analysis. European Journal of Operational Research, 80(3), 588-607.

Thanassoulis, E., Portela, M.C.S., Despić, O. (2008) Data envelopment analysis: The mathematical programming approach to efficiency analysis. In H.O. Fried, C.A.K Lovell, S.S. Schmidt (Eds.), The measurement of productive efficiency and productivity growth (pp. 251-420). New York: Oxford University Press. 